\title{
Bipartite and neighborhood graphs and the spectrum of the normalized graph Laplace operator
}

Frank BaUer AND JÜrgen Jost

\begin{abstract}
We study the spectrum of the normalized Laplace operator of a connected graph $\Gamma$. As is well known, the smallest non-trivial eigenvalue measures how difficult it is to decompose $\Gamma$ into two large pieces, whereas the largest eigenvalue controls how close $\Gamma$ is to being bipartite. The smallest eigenvalue can be controlled by the Cheeger constant, and we establish a dual construction that controls the largest eigenvalue. Moreover, we find that the neighborhood graphs $\Gamma[l]$ of order $l \geq 2$ encode important spectral information about $\Gamma$ itself which we systematically explore. In particular, the neighborhood graph method leads to new estimates for the smallest non-trivial eigenvalue that can improve the Cheeger inequality, as well as an explicit estimate for the largest eigenvalue from above and below. As applications of such spectral estimates, we provide a criterion for the synchronizability of coupled map lattices, and an estimate for the convergence rate of random walks on graphs.
\end{abstract}

\section{Introduction}

2 The graph Laplace operator and its basic properties

3 The Cheeger constant and its dual and eigenvalue estimates 795

4 Relations between $h$ and $\bar{h}$

$5 \quad$ Neighborhood graphs

6 Comparison of the Cheeger estimates with the estimates obtained by the neighborhood graph method 
8 Controlling the largest eigenvalue in terms of a local clustering coefficient
9 Random walks on graphs and the convergence to equilibrium

10 Synchronization in coupled map lattices

Acknowledgments

References

842

\section{Introduction}

A general principle in geometry tells us that the spectrum of a Laplace operator encodes important geometric information about the underlying space. This principle has been particularly fertile in Riemannian geometry. One of the key questions has been the control from below of the first non-zero eigenvalue of the Laplace-Beltrami operator in terms of the geometry of the underlying Riemannian manifold (assumed to be compact here for simplicity of exposition). The Lichnerowicz bound estimates the first eigenvalue from below in terms of a lower bound for the Ricci curvature. In contrast, the Cheeger estimate controls the first eigenvalue from below in terms of a global quantity that expresses how difficult it is to cut the manifold into two large pieces [8]. In this way, the first eigenvalue could be related to the fundamental analytic constants of a Riemannian manifold, like the isoperimetric or Sobolev constants. The work of Li and Yau [24] utilized gradient bounds for eigenfunctions in order to control the first eigenvalue from below in terms of the diameter and Ricci bounds of the Riemannian manifold. More generally, their famous Harnack inequality for the heat kernel [25] then allowed for a systematic control of all eigenvalues of a Riemannian manifold, with the optimal asymptotics as given by Weyl's law. See for instance [7] for a systematic treatment of eigenvalues in Riemannian geometry.

In graph theory, the algebraic graph Laplace operator has been explored for a long time; see for example [26]. More recently, Chung and Yau, see e.g., [11-13] and the monograph [10], systematically investigated the normalized graph Laplace operator $\Delta$ of an unweighted and undirected graph. This operator, which is different from the algebraic graph Laplace operator, underlies random walks and diffusion processes with conservation laws on graphs. The normalized graph Laplace operator is related to the Laplace-Beltrami 
operator for a Riemannian manifold. Thus, in order to study the spectrum of $\Delta$, one can systematically apply methods developed in Riemannian geometry for the investigation of the spectrum of the Laplace-Beltrami operator and this led to many remarkable insights, see the works just cited and the references therein.

In particular, the smallest non-trivial eigenvalue can be well controlled in terms of the Cheeger constant [10]. (In a graph-theoretical setting, such constants can already be found in earlier work by Polyá and Szegö [29].)

In contrast to a Riemannian manifold, on a graph, the spectrum of the normalized Laplace operator is always bounded from above. In fact, the upper bound 2 is achieved if and only if the graph is bipartite. (We recall that a graph is bipartite if its vertex set consists of two classes such that edges are only permitted between two vertices from opposite classes.) Therefore, it is a natural question how to control the largest eigenvalue for graphs that are not bipartite. The original goal of this article was to derive bounds for the largest eigenvalue of $\Delta$ from above and below. These bounds reflect how different the graph in question is from a bipartite one resp. how close it is to such a graph.

In fact, however, these estimates led us to discover more general structures that go beyond our original goal. First of all, we construct a dual version $\bar{h}$ of the Cheeger constant $h$ and derive bounds for the largest eigenvalue from above and below in terms of $\bar{h}$. We find interesting relations between $h$ and $\bar{h}$, and the combination of these two constants tells us more about the graph than either of them does individually. Moreover, we find that the neighborhood graphs $\Gamma[l]$, of order $l \geq 2$, of a graph $\Gamma$ also encode important spectral information about $\Gamma$ itself. For concreteness let $l=2$ for the moment. The idea then is that $\Gamma[2]$ is a weighted graph with the same vertices as $\Gamma$ itself, and two vertices are connected in $\Gamma[2]$ when they share at least one neighbor in $\Gamma$, with lower weights for more shared neighbors. More precisely, let $\Gamma$ be a weighted graph, with the weight of the edge between the vertices $i$ and $j$ denoted by $w_{i j}$ (which is 0 unless $i$ and $j$ are neighbors), and the degree of $i$ being $d_{i}=\sum_{j} w_{i j}$. For the neighborhood graph $\Gamma[2]$, the weight of the edge $e[2]=(i, j)$ in $\Gamma[2]$ then is given by $w_{i j}[2]=\sum_{k} \frac{1}{d_{k}} w_{i k} w_{k j}$. Consequently, $i$ and $j$ are neighbors in $\Gamma[2]$ if $i$ and $j$ have at least one common neighbor in $\Gamma$, i.e., there exists a path of length 2 between $i$ and $j$ in $\Gamma$. Note that the weights of $\Gamma[2]$ are normalized in such a manner that every vertex $i$ has the same degree in both $\Gamma$ and $\Gamma[2]$. It turns out that the eigenvalues of $\Gamma[2]$ are given by $\lambda(2-\lambda)$ when $\lambda$ stands for the eigenvalues of $\Gamma$. This well suits our purpose because controlling the highest eigenvalue $\lambda_{\max }$ from above is equivalent to controlling $2-\lambda_{\max }$ from below. Thus, lower 
spectral bounds on $\Gamma[2]$ yield lower and upper spectral bounds for $\Gamma$. For certain graphs, these new lower bounds improve the Cheeger estimate for the smallest non-zero eigenvalue. We not only utilize this principle to derive such bounds, but we also explore the relation between the spectra of $\Gamma$ and $\Gamma[2]$ in more general terms. Naturally, the construction of the neighborhood graph $\Gamma[2]$ can be generalized to higher order neighborhood graphs, i.e., $i$ and $j$ are neighbors in $\Gamma[l]$ if there exists a path of length $l$ between $i$ and $j$ in $\Gamma$. Again, the weights of $\Gamma[l]$ are normalized in such a way that every vertex has the same degree in both $\Gamma$ and $\Gamma[l]$. In the present paper, we also explore the spectra of the higher order neighborhood graphs $\Gamma[l]$ and their relations to the spectrum of $\Gamma$. The concept of the neighborhood graph is quite general, and can also be used to investigate the spectrum of the normalized graph Laplace operator defined on directed [4] graphs.

In the last two sections, we will apply our new eigenvalue bounds to two concrete problems - the convergence of random walks on graphs, and the synchronization for coupled map lattices, that is, a dynamical system supported on the vertices of a graph and coupled according to the interaction structure given by the edges of the graph. Again, the principle is that eigenvalue estimates control how different the graph in question is from the two extremes of a disconnected or a bipartite graph. On a disconnected or a bipartite graph, for different reasons, the random walk does not converge to a stationary distribution, and the coupled map lattice does not synchronize.

\section{The graph Laplace operator and its basic properties}

In this paper, $\Gamma$ is an undirected, weighted, connected, finite graph of $N$ vertices. We do not exclude loops, i.e., edges connecting a vertex with itself. The vertices are denoted by $i, j, \ldots . V$ denotes the vertex and $E$ the edge set of $\Gamma$, respectively. When the vertices $i$ and $j$ are connected by an edge, they are called neighbors, in symbols $i \sim j$. The associated weight function $w: V \times V \rightarrow \mathbb{R}$ satisfies $w_{i j}=w_{j i}$ and $w_{i j}>0$ whenever $i \sim j$ and $w_{i j}=0$ iff $i \nsim j$. For a vertex $i$, its degree $d_{i}$ is given by $d_{i}:=\sum_{j} w_{i j}$. When $w_{i j}=1$ whenever $i \sim j$, we shall speak of an unweighted graph.

The clustering coefficient $C$ of an unweighted graph $\Gamma$ is defined as

$$
C:=\frac{3 \times \text { number of triangles }}{\text { number of connected triples of vertices }},
$$

where a triangle is a triple of mutually connected vertices. The clustering coefficient measures how many connections there exist between the neighbors of a node. $C$ becomes maximal if $\Gamma$ is a fully connected graph. In contrast, $C$ 
vanishes when $\Gamma$ is a bipartite graph, that is, consists of two classes $V_{1}, V_{2}$ of vertices such that no vertices in the same class are connected by an edge. In particular, there are no loops in a bipartite graph. Equivalently, a graph is bipartite iff it has no cycles of odd length, and thus in particular no triangles.

We now recall the definition of the normalized graph Laplace operator and state its basic properties.

Definition 2.1. We have a natural measure $\mu$ on the vertex set $V$ given by $\mu(i)=d_{i}$. The inner product of two functions $u, v \in \ell^{2}(V, \mu)$ is defined as

$$
(u, v)_{\mu}:=\sum_{i \in V} \mu(i) u(i) v(i) .
$$

The Hilbertspace $\ell^{2}(V, \mu)$ is then given by

$$
\ell^{2}(V, \mu)=\left\{u: V \rightarrow \mathbb{R} \quad \mid \quad(u, u)_{\mu}<\infty\right\}
$$

Since we consider only finite graphs here, the space $\ell^{2}(V, \mu)$ is nothing but the space of real-valued functions on $V$ endowed with the inner product $(\cdot, \cdot)_{\mu}$. We study the normalized graph Laplace operator

$$
\begin{gathered}
\Delta: \ell^{2}(V, \mu) \rightarrow \ell^{2}(V, \mu) \\
\Delta v(i):=\frac{1}{d_{i}}\left(\sum_{j} w_{i j}(v(i)-v(j))\right) .
\end{gathered}
$$

The Laplace operator $\Delta$ underlies random walks on graphs. In fact, the Laplace operator $\Delta$ can be considered as $\Delta=: I-P$, where $I$ denotes the identity and $P$ is transition probability operator of a random walk (or sometimes called the Markov operator), respectively. We should point our here that the normalized graph Laplace operator $\Delta$ is not exactly the one studied by Chung [10]. However, both Laplace operators are unitarily equivalent and therefore have the same spectrum. We recall the following basic properties:

(i) $\Delta$ is self-adjoint w.r.t. $(., .)_{\mu}$, i.e.,

$$
(u, \Delta v)_{\mu}=(\Delta u, v)_{\mu}
$$

for all $u, v \in \ell^{2}(V, \mu)$. This follows from the symmetric weight function, i.e., $w_{i j}=w_{j i}$ for all $i$ and $j$. 
Moreover,

(ii) $\Delta$ is non-negative, i.e.,

$$
(\Delta u, u)_{\mu} \geq 0
$$

for all $u \in \ell^{2}(V, \mu)$. This follows from the Cauchy-Schwarz inequality.

(iii) $\Delta u=0$ iff $u$ is constant.

Clearly, $\Delta u=0$ if $u$ is constant. Let $\Delta u=0$ and assume that $u$ is not constant. Then there exists a vertex, say $i$, with $u(i) \geq u(j)$ for all $j \sim i$ with strict inequality for at least one such $j$. Thus there exists a non-trivial local maximum. This is a contradiction since $\Delta u(i)=0$ implies that the value $u(i)$ is the average of the values at the neighbors of $i$. Since $\Gamma$ is connected, $u$ then has to be a constant. (When $\Gamma$ is not connected, a solution of $\Delta u=0$ is constant on every connected component of $\Gamma$.)

We say that $\lambda$ is an eigenvalue of $\Delta$ if there exists some $u \not \equiv 0$ with

$$
\Delta u=\lambda u \text {. }
$$

The preceding properties have consequences for the eigenvalues of $\Delta$ :

- By (i), the eigenvalues are real.

- By (ii), they are non-negative, i.e., $\lambda_{k} \geq 0$ for all $k$.

- By (iii), the smallest eigenvalue is $\lambda_{0}=0$. Since $\Gamma$ is connected, this eigenvalue is simple, i.e.,

$$
\lambda_{k}>0
$$

for $k>0$ where the eigenvalues are ordered as

$$
\lambda_{0}=0<\lambda_{1} \leq \cdots \leq \lambda_{N-1}
$$

In the literature, the normalized Laplace operator $\Delta$ is sometimes introduced in a different way (see for instance [34]): let $\mathcal{E}$ be the set of all oriented edges in $\Gamma$. We denote by $o(e)$ the origin and by $t(e)$ the terminus of the edge $e \in \mathcal{E}$, respectively. Furthermore, $\bar{e}$ denotes the inversion of the edge $e \in \mathcal{E}$. Let $C^{0}(V, \mathbb{R})=\{u: V \rightarrow \mathbb{R}\}$ and $C^{1}(V, \mathbb{R})=\{f: E \rightarrow \mathbb{R}, f(\bar{e})=-f(e)\}$ be 
the 0th and 1st cochain group and let

$$
(u, v)_{\mu}=\sum_{i \in V} \mu(i) u(i) v(i)
$$

and

$$
(f, g)_{\mu}=\frac{1}{2} \sum_{e \in \mathcal{E}} \mu(e) f(e) g(e)
$$

be inner products on $C^{0}(V, \mathbb{R})$ and $C^{1}(V, \mathbb{R})$, respectively, where $\mu(i)=d_{i}$ and $\mu(e)=w_{i j}$ for $e=(i, j) \in \mathcal{E}$. Note that $C^{0}(V, \mathbb{R})$ together with the inner product $(2.8)$ is equal to $\ell^{2}(V, \mu)$. Now we can define the normalized Laplace operator as

$$
\begin{gathered}
\Delta: C^{0}(V, \mathbb{R}) \rightarrow C^{0}(V, \mathbb{R}), \\
\Delta=d^{*} d
\end{gathered}
$$

where $d: C^{0}(V, \mathbb{R}) \rightarrow C^{1}(V, \mathbb{R})$,

$$
(d v)(e)=v(t(e))-v(o(e))
$$

is the coboundary operator ( $d$ can be considered as a discrete analog of the exterior derivative) and $d^{*}: C^{1}(V, \mathbb{R}) \rightarrow C^{0}(V, \mathbb{R})$

$$
\left(d^{*} f\right)(i)=-\frac{1}{\mu(i)} \sum_{e \in \mathcal{E}_{i}} \mu(e) f(e)
$$

is the (formal) adjoint of $d$ with respect to the inner products (2.8) on $C^{0}(V, \mathbb{R})$ and $(2.9)$ on $C^{1}(V, \mathbb{R})$. Here, $\mathcal{E}_{i}$ is given by $\mathcal{E}_{i}:=\{e \in \mathcal{E}: o(e)=i\}$.

A simple calculation shows that

$$
\begin{aligned}
(d u, d v)_{\mu} & =\frac{1}{2} \sum_{e=(i, j) \in \mathcal{E}} w_{i j}(u(i)-u(j))(v(i)-v(j)) \\
(2.12) & =\frac{1}{2}\left(\sum_{i, j \in V} w_{i j} u(i) v(i)+\sum_{i, j \in V} w_{i j} u(j) v(j)-2 \sum_{i, j \in V} w_{i j} u(i) v(j)\right) \\
& =\sum_{i \in V} d_{i} u(i)\left(v(i)-\frac{1}{d_{i}} \sum_{j \in V} w_{i j} v(j)\right) \\
& =(u, \Delta v)_{\mu} .
\end{aligned}
$$


This confirms that the two definitions (2.3) and (2.10) of the normalized Laplace operator coincide.

An orthonormal basis of $\ell^{2}(V, \mu)$ consisting of eigenfunctions of $\Delta$,

$$
u_{k}, k=0, \ldots, N-1
$$

can be constructed in the standard way, which we now recall. Let $H_{0}:=$ $H:=\ell^{2}(V, \mu)$ be the Hilbert space of all real-valued functions on $\Gamma$ with the inner product $(., .)_{\mu}$. We iteratively define

$$
H_{k}:=\left\{v \in H:\left(v, u_{i}\right)_{\mu}=0 \text { for } i \leq k-1\right\},
$$

starting with a constant function $u_{0}$ as the eigenfunction for the eigenvalue $\lambda_{0}=0$. Then the $k$ th eigenvalue is given by

$$
\lambda_{k}=\inf _{u \in H_{k}-\{0\}} \frac{(d u, d u)_{\mu}}{(u, u)_{\mu}},
$$

and the corresponding eigenfunction $u_{k}$ realizes this infimum. By way of contrast, the highest eigenvalue is also given by

$$
\lambda_{N-1}=\sup _{u \neq 0} \frac{(d u, d u)_{\mu}}{(u, u)_{\mu}} .
$$

In particular, for any eigenfunction $u$ for some eigenvalue $\lambda$, we then have

$$
\lambda=\frac{(d u, d u)_{\mu}}{(u, u)_{\mu}} .
$$

All different eigenfunctions are orthogonal to each other. In particular the eigenfunctions $u_{1}, \ldots u_{N-1}$ are orthogonal to $u_{0}$, the eigenfunction for the eigenvalue $\lambda_{0}=0$. This implies that

$$
\sum_{i} d_{i} u_{k}(i)=0
$$

for $k=1, \ldots, N-1$, since $u_{0}(i)$ is constant for all $i$.

The largest eigenvalue satisfies

$$
\lambda_{N-1} \leq 2
$$

with equality if and only if $\Gamma$ is bipartite. A corresponding eigenfunction equals a positive constant $c$ on one class and $-c$ on the other class of vertices. In contrast, for loopless graphs, the highest eigenvalue $\lambda_{N-1}$ becomes 
smallest on a complete graph $K_{N},{ }^{1}$ namely

$$
\lambda_{N-1}=\frac{N}{N-1}
$$

By considering the trace of $\Delta$ we obtain

$$
\sum_{i} \lambda_{i}=N-\sum_{i} \frac{w_{i i}}{d_{i}}
$$

Altogether, the eigenvalues satisfy

$$
0=\lambda_{0}<\lambda_{1} \leq \frac{N-\sum_{i} \frac{w_{i i}}{d_{i}}}{N-1} \leq \lambda_{N-1} \leq 2
$$

Similarly to (2.20), the first eigenvalue $\lambda_{1}$ is largest for the complete graph $K_{N}$, achieving the bound in $(2.22)$, that is

$$
\lambda_{1}=\frac{N}{N-1} .
$$

For any other unweighted graph, we have in fact

$$
\lambda_{1} \leq 1
$$

Hence, the complete graph $K_{N}$ satisfies

$$
\lambda_{0}=0 \text { and } \lambda_{1}=\cdots=\lambda_{N-1}=\frac{N}{N-1} .
$$

In fact, it is easy to show that for the complete graph $K_{N}$ any function $v$ that satisfies $\sum_{j} d_{j} v(j)=0$ is an eigenfunction for the eigenvalue $\frac{N}{N-1}$.

\section{The Cheeger constant and its dual and eigenvalue estimates}

Our starting point are the estimates for the first eigenvalue $\lambda_{1}$ in terms of the (Polya-)Cheeger constant, see for example $[1,2,9,10,17]$. The (Polya-)

\footnotetext{
${ }^{1} K_{N}$ denotes an unweighted complete loopless graph on $N$ vertices.
} 
Cheeger constant [29] of a weighted graph is defined as

$$
h:=\min _{U} \frac{|E(U, \bar{U})|}{\min \{\operatorname{vol}(U), \operatorname{vol}(\bar{U})\}}=\min _{U \subset V: \operatorname{vol}(U) \leq \frac{1}{2} \operatorname{vol}(V)} \frac{|E(U, \bar{U})|}{\operatorname{vol}(U)},
$$

where $U$ and $\bar{U}=V \backslash U$ yield a partition of the vertex set $V$ and $U, \bar{U}$ are both non-empty. Here, the volume of $U$ is given by $\operatorname{vol}(U):=\sum_{i \in U} d_{i}$, $E(U, \bar{U}) \subseteq E$ is the subset of all edges with one vertex in $U$ and one vertex in $\bar{U}$, and $|E(U, \bar{U})|:=\sum_{k \in U, l \in \bar{U}} w_{k l}$ is the sum of the weights of all edges in $E(U, \bar{U})$. In general, we have $h \leq 1$ and equality holds for instance if $\Gamma$ is given by $K_{2}$ or $K_{3}$. This follows from the definition of $h$, since $|E(U, \bar{U})| \leq$ $|E(U, \bar{U})|+|E(U, U)|=\operatorname{vol}(U)$ and $|E(U, \bar{U})| \leq|E(U, \bar{U})|+|E(\bar{U}, \bar{U})|=$ $\operatorname{vol}(\bar{U})$. Let us first recall $[9,10]$ how $h$ can bound $\lambda_{1}$ from above. We use the variational characterization (2.15), observing that $H_{1}$ is the set of all functions $v$ with the normalization $\sum_{i \in V} d_{i} v(i)=0$. Let the edge set $E(U, \bar{U})$ divide the graph into the two disjoint sets $U, \bar{U}$ of nodes, and let $U$ be the one with the smaller volume $\operatorname{vol}(U)=\sum_{i \in U} d_{i}$. We consider a function $v$ that is $=1$ on all the nodes in $U$ and $=-\alpha$ for some positive $\alpha$ on $\bar{U}$. $\alpha$ is chosen so that the normalization $\sum_{i \in V} d_{i} v(i)=0$ holds, that is, $\sum_{i \in U} d_{i}-\sum_{i \in \bar{U}} d_{i} \alpha=0$. Since $\bar{U}$ is the subset with the larger volume $\sum_{i \in \bar{U}} d_{i}$, we have $\alpha \leq 1$. Thus, for our choice of $v$, the quotient in (2.15) becomes $\leq \frac{(1+\alpha)^{2}|E(U, \bar{U})|}{\sum_{i \in U} d_{i}+\sum_{i \in \bar{U}} d_{i} \alpha^{2}}=\frac{(1+\alpha)|E(U, \bar{U})|}{\sum_{i \in U} d_{i}} \leq 2 \frac{|E(U, \bar{U})|}{\sum_{i \in U} d_{i}}=2 \frac{|E(U, \bar{U})|}{\operatorname{vol}(U)}$. Since this holds for all such splittings of our graph $\Gamma$, we obtain from (3.1) and (2.15)

$$
\lambda_{1} \leq 2 h .
$$

As a lower bound for $\lambda_{1}$ in terms of the Cheeger constant $h$ we obtain:

$$
\lambda_{1} \geq 1-\sqrt{1-h^{2}} .
$$

In fact, the estimates (3.2) and (3.3) hold under rather general conditions, and an appropriate version is also true for the algebraic (non-normalized) graph Laplace operator [27].

The crucial step in the proof of (3.3) is the next lemma which we recall here, because we will make use of it in the following. The proof given here 
is mainly based on [16] and uses some generalizations that can be found in $[10]$.

Lemma 3.1. Let $g \in \ell^{2}(V, \mu)$ with $S(g):=\{i \in V: g(i)>0\} \neq \emptyset$, put

$$
h(g):=\min _{\emptyset \neq S \subseteq S(g)} \frac{|E(S, \bar{S})|}{\operatorname{vol}(S)}
$$

and let $g_{+}$be the positive part of $g$, i.e.,

$$
g_{+}(i)=\left\{\begin{array}{ccc}
g(i) & \text { if } & g(i)>0 \\
0 & \text { else }
\end{array}\right.
$$

Then

$$
1+\sqrt{1-h^{2}(g)} \geq \frac{\sum_{e=(i, j)} w_{i j}\left(g_{+}(i)-g_{+}(j)\right)^{2}}{\sum_{i} d_{i} g_{+}(i)^{2}} \geq 1-\sqrt{1-h^{2}(g)}
$$

Proof. For technical reasons it is convenient to define the new weights $\mu_{i j}$ by $\mu_{i j}=w_{i j}$, for all $i \neq j$ and $\mu_{i i}=\frac{1}{2} w_{i i}$ for all $i \in V$.

First, we write

$$
\begin{aligned}
W & :=\frac{\sum_{e=(i, j)} w_{i j}\left(g_{+}(i)-g_{+}(j)\right)^{2}}{\sum_{i} d_{i} g_{+}(i)^{2}} \\
& =\frac{\sum_{e=(i, j)} \mu_{i j}\left(g_{+}(i)-g_{+}(j)\right)^{2}}{\sum_{i} d_{i} g_{+}(i)^{2}} \\
& =\frac{\sum_{e=(i, j)} \mu_{i j}\left(g_{+}(i)-g_{+}(j)\right)^{2} \sum_{e=(i, j)} \mu_{i j}\left(g_{+}(i)+g_{+}(j)\right)^{2}}{\sum_{i} d_{i} g_{+}(i)^{2} \sum_{e=(i, j)} \mu_{i j}\left(g_{+}(i)+g_{+}(j)\right)^{2}} \\
& =: \frac{I}{I I} .
\end{aligned}
$$

Using the Cauchy-Schwarz inequality we obtain

$$
I \geq\left(\sum_{e=(i, j)} \mu_{i j}\left|g_{+}(i)^{2}-g_{+}(j)^{2}\right|\right)^{2}=\left(\sum_{e=(i, j)} w_{i j}\left|g_{+}(i)^{2}-g_{+}(j)^{2}\right|\right)^{2}
$$


Now we have

$$
\begin{aligned}
\sum_{e=(i, j)} w_{i j}\left|g_{+}(i)^{2}-g_{+}(j)^{2}\right| & =\sum_{e=(i, j): g_{+}(i)>g_{+}(j)} w_{i j}\left(g_{+}(i)^{2}-g_{+}(j)^{2}\right) \\
& =2 \sum_{e=(i, j): g_{+}(i)>g_{+}(j)} w_{i j} \int_{g_{+}(j)}^{g_{+}(i)} t d t \\
& =2 \int_{0}^{\infty} \sum_{e=(i, j): g_{+}(j) \leq t<g_{+}(i)} w_{i j} t d t .
\end{aligned}
$$

Note that $\sum_{e=(i, j): g_{+}(j) \leq t<g_{+}(i)} w_{i j}=\left|E\left(S_{t}, \overline{S_{t}}\right)\right|$ where $S_{t}:=\left\{i: g_{+}(i)>t\right\}$. Using (3.4) we obtain,

$$
\begin{aligned}
\sum_{e=(i, j)} w_{i j}\left|g_{+}(i)^{2}-g_{+}(j)^{2}\right| & \geq 2 h(g) \int_{0}^{\infty} \operatorname{vol}\left(S_{t}\right) t d t \\
& =2 h(g) \int_{0}^{\infty} \sum_{i: g_{+}(i)>t} d_{i} t d t \\
& =2 h(g) \sum_{i \in V} d_{i} \int_{0}^{g_{+}(i)} t d t \\
& =h(g) \sum_{i} d_{i} g_{+}(i)^{2}
\end{aligned}
$$

and so it follows:

$$
\begin{gathered}
I \geq h^{2}(g)\left(\sum_{i} d_{i} g_{+}(i)^{2}\right)^{2} \cdot \\
I I=\sum_{i} d_{i} g_{+}(i)^{2} \sum_{e=(i, j)} \mu_{i j}\left(g_{+}(i)+g_{+}(j)\right)^{2} \\
=\sum_{i} d_{i} g_{+}(i)^{2}\left(\sum_{i} d_{i} g_{+}(i)^{2}+\sum_{i, j} w_{i j} g_{+}(i) g_{+}(j)\right) \\
=\sum_{i} d_{i} g_{+}(i)^{2}\left(2 \sum_{i} d_{i} g_{+}(i)^{2}-\sum_{e=(i, j)} \mu_{i j}\left(g_{+}(i)-g_{+}(j)\right)^{2}\right) \\
=(2-W)\left(\sum_{i} d_{i} g_{+}(i)^{2}\right)^{2} .
\end{gathered}
$$


Combining everything we obtain,

$$
W \geq \frac{h^{2}(g)}{(2-W)}
$$

and consequently

$$
1+\sqrt{1-h^{2}(g)} \geq W \geq 1-\sqrt{1-h^{2}(g)} .
$$

The second observation that we need to prove the Cheeger inequality (3.3) is the following lemma [16]:

Lemma 3.2. For every non-negative real number $\lambda$ and $g \in \ell^{2}(V, \mu)$ we have

$$
\lambda \geq \frac{\sum_{e=(i, j)} w_{i j}\left(g_{+}(i)-g_{+}(j)\right)^{2}}{\sum_{i} d_{i} g_{+}(i)^{2}}=W
$$

if $\Delta g(i) \leq \lambda g(i)$ for all $i \in S(g)$.

Proof. We have

$$
\left(\Delta g, g_{+}\right)_{\mu}=\sum_{i \in V} d_{i} \Delta g(i) g_{+}(i) \leq \lambda \sum_{i \in S(g)} d_{i} g_{+}(i) g_{+}(i)=\lambda \sum_{i \in V} d_{i} g_{+}(i) g_{+}(i)
$$

and

$$
\begin{aligned}
\left(\Delta g, g_{+}\right)_{\mu} & =\left(d g, d g_{+}\right)_{\mu}=\sum_{e=(i, j) \in E} w_{i j}(g(i)-g(j))\left(g_{+}(i)-g_{+}(j)\right) \\
& \geq \sum_{e=(i, j) \in E} w_{i j}\left(g_{+}(i)-g_{+}(j)\right)^{2} .
\end{aligned}
$$

The Cheeger inequality now follows from the last two lemmata by taking $\lambda=$ $\lambda_{1}$ and $g=u_{1}$ an eigenfunction for $\lambda_{1}$. Since $\left(u_{1}, \mathbb{1}\right)_{\mu}=0$, we have $S\left(u_{1}\right) \neq \emptyset$ and $T\left(u_{1}\right):=\left\{i \in V: u_{1}(i)<0\right\} \neq \emptyset$. This implies that it is always possible to choose $u_{1}$ such that $\operatorname{vol}\left(S\left(u_{1}\right)\right) \leq \operatorname{vol}\left(\overline{S\left(u_{1}\right)}\right)$ (if $\operatorname{vol}\left(S\left(u_{1}\right)\right) \geq \operatorname{vol}\left(\overline{S\left(u_{1}\right)}\right)$ take $-u_{1}$ instead of $\left.u_{1}\right)$ and thus $h\left(u_{1}\right) \geq h$.

In any case, in qualitative terms, the Cheeger inequalities (3.2) and (3.3) simply say that $\lambda_{1}$ becomes small when the graph can be easily (that is, by cutting only few edges) decomposed into two large parts. Thus, $\lambda_{1}$ is small, 
that is, close to its minimal value 0 , when $\Gamma$ is similar to a disconnected graph, with equality iff $\Gamma$ is disconnected itself. Similarly, and this brings us to our topic, the largest eigenvalue is large, that is, close to its maximal value 2 , when $\Gamma$ is close to a bipartite graph, with equality iff $\Gamma$ is bipartite itself.

The main purpose of this section then is a dual version of (3.2) and (3.3) for the largest eigenvalue $\lambda_{N-1}$. More precisely, we shall obtain an estimate for $\lambda_{N-1}$ in terms of a dual version of the Cheeger constant which we now introduce. Let $V_{1}, V_{2}$ and $\overline{V_{1} \cup V_{2}}=: V_{3}$ be a partition of the vertex set $V$ into three disjoint sets such that $V_{1}$ and $V_{2}$ are non-empty.

It is helpful to think of $V_{1} \cup V_{2}$ as the (almost) bipartite part of $\Gamma$ and $V_{3}$ as the part of $\Gamma$ that contains many cycles of odd length, i.e., $V_{3}$ is not bipartite.

For a partition $V_{1}, V_{2}, V_{3}$ of the vertex set $V$ we define:

$$
\bar{h}:=\max _{V_{1}, V_{2}} \frac{2\left|E\left(V_{1}, V_{2}\right)\right|}{\operatorname{vol}\left(V_{1}\right)+\operatorname{vol}\left(V_{2}\right)},
$$

where as before the volume of $V_{k}$ is given by $\operatorname{vol}\left(V_{k}\right):=\sum_{i \in V_{k}} d_{i}$ and $\mid E\left(V_{i}\right.$, $\left.V_{j}\right) \mid:=\sum_{k \in V_{i}, l \in V_{j}} w_{k l}$.

The next theorem shows that $\bar{h}$ characterizes bipartite graphs.

Theorem 3.1. $\bar{h} \leq 1$, and $\bar{h}=1$ if and only if $\Gamma$ is bipartite.

Proof. First, note that, for a partition $V_{1}, V_{2}$ and $V_{3}$ of $V$, the volume of $V_{i}$ can also be written in the form

$$
\operatorname{vol}\left(V_{i}\right)=\sum_{j=1}^{3}\left|E\left(V_{i}, V_{j}\right)\right|
$$

Consequently, $\bar{h}$ is given by

$$
\bar{h}=\max _{V_{1}, V_{2}} \frac{2\left|E\left(V_{1}, V_{2}\right)\right|}{\sum_{j=1}^{3}\left|E\left(V_{1}, V_{j}\right)\right|+\sum_{j=1}^{3}\left|E\left(V_{2}, V_{j}\right)\right|} .
$$

Thus, clearly,

$$
\bar{h} \leq 1
$$

Assume that $\Gamma$ is bipartite. Then there exists a partition $V_{1}, V_{2}, V_{3}$ of $V$ such that $V_{3}=\emptyset$ and there are no edges within the subsets $V_{1}$ and $V_{2}$. 
For this partition, it follows that $\left|E\left(V_{1}, V_{3}\right)\right|=\left|E\left(V_{2}, V_{3}\right)\right|=\left|E\left(V_{1}, V_{1}\right)\right|=$ $\left|E\left(V_{2}, V_{2}\right)\right|=0$. By (3.7) this implies that $\bar{h} \geq 1$. Together with (3.8) it follows that $\bar{h}=1$.

Now assume that $\bar{h}=1$. Equation (3.7) implies that there exists a partition $V_{1}, V_{2}, V_{3}$ of $V$ such that $\left|E\left(V_{1}, V_{3}\right)\right|=\left|E\left(V_{2}, V_{3}\right)\right|=\left|E\left(V_{1}, V_{1}\right)\right|=$ $\left|E\left(V_{2}, V_{2}\right)\right|=0$. Since $\Gamma$ is connected $V_{3}=\emptyset$ and thus $\Gamma$ is bipartite.

As an illustration, let us consider loopless Erdös-Renyi random graphs, i.e., we start with a given vertex set and add edges between two vertices with a fixed probability $p$. If we start with $p=1$ then we obtain a complete graph and thus $\bar{h} \approx 1 / 2$, as will be shown in Example 4.1. Now if we decrease $p$ we decrease the number of edges in the graph. This will lead to a local bipartite subgraph in $\Gamma$ and thus $\bar{h}$ will be increased. If we decrease $p$ further we finally have $|E| \approx|V|$, i.e., the graph will be approximately a tree (we assume that the random graphs are connected) thus $\bar{h} \approx 1$. We conclude that, for random graphs $\bar{h}$ is a function of $p$. More details are revealed by numerical simulations.

Proposition 3.1. For a loopless graph $\Gamma$,

$$
\frac{1}{2} \leq \bar{h}
$$

Proof. Assume that there exists a partition $V_{1}, V_{2}$ and $V_{3}=\emptyset$ of the vertex set $V$ such that

$$
\left|E\left(V_{1}, V_{2}\right)\right| \geq \max _{i=1,2}\left|E\left(V_{i}, V_{i}\right)\right|
$$

Then by using (3.6) we obtain:

$$
\begin{aligned}
\bar{h} & \geq \max _{V_{1}, V_{2}, V_{3}=\emptyset} \frac{2\left|E\left(V_{1}, V_{2}\right)\right|}{2\left|E\left(V_{1}, V_{2}\right)\right|+\left|E\left(V_{1}, V_{1}\right)\right|+\left|E\left(V_{2}, V_{2}\right)\right|} \\
& \geq \max _{V_{1}, V_{2}, V_{3}=\emptyset} \frac{\left|E\left(V_{1}, V_{2}\right)\right|}{\left|E\left(V_{1}, V_{2}\right)\right|+\max _{i=1,2}\left|E\left(V_{i}, V_{i}\right)\right|} \\
& \geq \frac{1}{2} .
\end{aligned}
$$

Thus, it is sufficient to find a partition that satisfies (3.9).

In the following, we will construct such a partition. Start with an arbitrarily partition $V_{1}, V_{2}$ and $V_{3}=\emptyset$ of $V$. If (3.9) is satisfied we are done. 
Otherwise, assume w.l.o.g. that $\left|E\left(V_{1}, V_{1}\right)\right|>\left|E\left(V_{1}, V_{2}\right)\right|$, i.e.,

$$
\sum_{i \in V_{1}} \sum_{j \in V_{1}} w_{i j}>\sum_{i \in V_{1}} \sum_{j \in V_{2}} w_{i j} .
$$

We observe that there exists a vertex $i$ in $V_{1}$ such that

$$
\sum_{j \in V_{1}} w_{i j}>\sum_{j \in V_{2}} w_{i j}
$$

We remove the vertex $i$ from $V_{1}$ and add it to $V_{2}$. By doing so, $\left|E\left(V_{1}, V_{2}\right)\right|$ is increased by $\sum_{j \in V_{1}} w_{i j}-\sum_{j \in V_{2}} w_{i j}>0,\left|E\left(V_{1}, V_{1}\right)\right|$ is decreased by $\sum_{j \in V_{1}} w_{i j}$, and $\left|E\left(V_{2}, V_{2}\right)\right|$ is increased by $\sum_{j \in V_{2}} w_{i j}$. If (3.9) is still not satisfied, continue this procedure several times. Eventually, (3.9) holds since $\left|E\left(V_{1}, V_{2}\right)\right|$ is strictly monotonically increasing.

This lower bound is optimal, since Example 4.1 shows that for complete graphs $K_{N}, \bar{h}\left(K_{N}\right) \rightarrow \frac{1}{2}$ as $N \rightarrow \infty$. Clearly, the proof of Proposition 3.1 cannot be extended to graphs with loops. In fact, Proposition 3.1 only holds for loopless graphs, as can be seen by considering a graph with $\sum_{i} \frac{w_{i i}}{d_{i}}>$ $N-1$. In that case, $\bar{h} \geq 1 / 2$ would lead to a contradiction in Theorem 3.2 since

$$
1 \leq 2 \bar{h} \leq \lambda_{N-1}<1
$$

where we used (2.21) in the last inequality.

We now have a counterpart of the Cheeger inequality (3.2) and (3.3).

Theorem 3.2. The largest eigenvalue $\lambda_{N-1}$ of the graph Laplace operator $\Delta$ satisfies

$$
2 \bar{h} \leq \lambda_{N-1} \leq 1+\sqrt{1-(1-\bar{h})^{2}}
$$

Proof. First, we prove that $2 \bar{h} \leq \lambda_{N-1}$. The largest eigenvalue $\lambda_{N-1}$ of $\Delta$ is given by (2.16). Let $V_{1}, V_{2}, V_{3}$ be a partition that achieves $\bar{h}$. We consider the following function $u$ :

$$
u(i)=\left\{\begin{array}{lll}
\frac{1}{\operatorname{vol}\left(V_{1}\right)} & \text { if } & i \in V_{1}, \\
\frac{-1}{\operatorname{vol}\left(V_{2}\right)} & \text { if } & i \in V_{2} \\
0 & & \text { else. }
\end{array}\right.
$$


Substituting $u$ into (2.16) yields:

$$
\begin{aligned}
\lambda_{N-1}= & \sup _{v \neq 0} \frac{(d v, d v)_{\mu}}{(v, v)_{\mu}}=\sup _{v \neq 0} \frac{\sum_{e=(i, j)} w_{i j}(v(i)-v(j))^{2}}{\sum_{i} d_{i} v(i)^{2}} \\
\geq & \frac{\left(\frac{1}{\operatorname{vol}\left(V_{1}\right)}+\frac{1}{\operatorname{vol}\left(V_{2}\right)}\right)^{2}\left|E\left(V_{1}, V_{2}\right)\right|+\left(\frac{1}{\operatorname{vol}\left(V_{1}\right)}\right)^{2}\left|E\left(V_{1}, V_{3}\right)\right|+\left(\frac{1}{\operatorname{vol}\left(V_{2}\right)}\right)^{2}\left|E\left(V_{2}, V_{3}\right)\right|}{\left(\frac{1}{\operatorname{vol}\left(V_{1}\right)}+\frac{1}{\operatorname{vol}\left(V_{2}\right)}\right)} \\
\geq & \frac{\left(\operatorname{vol}\left(V_{1}\right)+\operatorname{vol}\left(V_{2}\right)\right)^{2}}{2 \operatorname{vol}\left(V_{1}\right) \operatorname{vol}\left(V_{2}\right)} \frac{2\left|E\left(V_{1}, V_{2}\right)\right|}{\operatorname{vol}\left(V_{1}\right)+\operatorname{vol}\left(V_{2}\right)} \\
& +\frac{\min \left(\operatorname{vol}\left(V_{1}\right), \operatorname{vol}\left(V_{2}\right)\right)}{\max \left(\operatorname{vol}\left(V_{1}\right), \operatorname{vol}\left(V_{2}\right)\right)} \frac{\left|E\left(V_{1} \cup V_{2}, V_{3}\right)\right|}{\left(\operatorname{vol}\left(V_{1}\right)+\operatorname{vol}\left(V_{2}\right)\right)} \\
\geq & 2 \bar{h}+\frac{\min \left(\operatorname{vol}\left(V_{1}\right), \operatorname{vol}\left(V_{2}\right)\right)}{\max \left(\operatorname{vol}\left(V_{1}\right), \operatorname{vol}\left(V_{2}\right)\right)} \frac{\left|E\left(V_{1} \cup V_{2}, V_{3}\right)\right|}{\left(\operatorname{vol}\left(V_{1}\right)+\operatorname{vol}\left(V_{2}\right)\right)} \\
\geq & 2 \bar{h},
\end{aligned}
$$

where we used the simple inequality $\frac{(a+b)^{2}}{2 a b} \geq 2$ for $a, b \in \mathbb{R}$.

Now we prove the remaining inequality $\lambda_{N-1} \leq 1+\sqrt{1-(1-\bar{h})^{2}}$. When one studies the largest eigenvalue of $\Delta$ it is convenient to introduce the operator $L=2 I-\Delta$. If $\lambda$ is an eigenvalue of $\Delta$ and corresponding eigenfunction $u$ then $u$ is also an eigenfunction for $L$ and corresponding eigenvalue $\mu=2-\lambda$. Thus, controlling the largest eigenvalue $\lambda_{N-1}$ of $\Delta$ from above is equivalent to controlling the smallest eigenvalue $\mu_{0}$ of $L$ from below. The smallest eigenvalue $\mu_{0}$ of $L$ is given by

$$
\begin{aligned}
\mu_{0} & =\inf _{u \neq 0} \frac{\frac{1}{2} \sum_{i, j \in V} w_{i j}(u(i)+u(j))^{2}}{\sum_{i \in V} d_{i} u(i)^{2}} \\
& =\inf _{u \neq 0} \frac{\sum_{e=(i, j) \in E} \mu_{i j}(u(i)+u(j))^{2}}{\sum_{i \in V} d_{i} u(i)^{2}}
\end{aligned}
$$

where as above $\mu_{i j}=w_{i j}$ for all $i \neq j$ and $\mu_{i i}=\frac{1}{2} w_{i i}$ for all $i \in V$. This simply follows from the standard min-max characterization of eigenvalues

$$
\mu_{0}=\inf _{u \neq 0} \frac{(L u, u)_{\mu}}{(u, u)_{\mu}} .
$$

We have for all $u, v \in \ell^{2}(V, \mu)$

$$
\begin{aligned}
(L u, v)_{\mu} & =\sum_{i} d_{i} L u(i) v(i)=\sum_{i} \sum_{j} w_{i j}(u(i)+u(j)) v(i) \\
& =\sum_{j} \sum_{i} w_{j i}(u(j)+u(i)) v(j)
\end{aligned}
$$


where we just exchanged $i$ and $j$. Adding the last two lines and setting $u=v$ yields

$$
(L u, u)_{\mu}=\frac{1}{2} \sum_{i, j} w_{i j}(u(i)+u(j))^{2} .
$$

In order to prove the lower bound for $\mu_{0}$ we will use a technique developed in [15]. The idea is the following: Construct a graph $\Gamma^{\prime}$ out of $\Gamma$ s.t. the quantity $h^{\prime}(g)$ defined in Lemma 3.1 for the new graph $\Gamma^{\prime}$ controls $\mu_{0}$ from below. In a second step, we show that $h^{\prime}(g)$ in turn can be controlled by the quantity $1-\bar{h}$ of the original graph. This then yields the desired estimate.

Let $u$ be an eigenfunction for the eigenvalue $\mu_{0}$ and define as above $S(u)=\{i \in V: u(i)>0\}$ and $T(u)=\{i \in V: u(i)<0\}$. Since $u$ is also an eigenfunction for $\lambda_{N-1}$ of $\Delta$ we know that $(u, \mathbb{1})_{\mu}=0$ and thus $S(u), T(u) \neq$ $\emptyset$. Then the new graph $\Gamma^{\prime}=\left(V^{\prime}, E^{\prime}\right)$ is constructed from $\Gamma$ in the following way. Duplicate all vertices in $S(u) \cup T(u)$ and denote the copies by a prime, e.g., if $i \in S(u)$ then the copy of $i$ is denoted by $i^{\prime}$. The copies of $S(u)$ and $T(u)$ are denoted by $S^{\prime}(u)$ and $T^{\prime}(u)$ respectively. The vertex set $V^{\prime}$ of $\Gamma^{\prime}$ is given by $V^{\prime}=V \cup S^{\prime}(u) \cup T^{\prime}(u)$. Every edge $(i, j) \in E(S(u), S(u))$ in $\Gamma$ is replaced by two edges $\left(i, j^{\prime}\right)$ and $\left(j, i^{\prime}\right)$ in $\Gamma^{\prime}$ s.t. $w_{i j}=w_{i j^{\prime}}^{\prime}=w_{j i^{\prime}}^{\prime}$. Similarly, if the edge is a loop, then $e=(i, i)$ is replaced by one edge $\left(i, i^{\prime}\right)$ s.t. $w_{i i}=w_{i i^{\prime}}$. The same is done with edges in $E(T(u), T(u))$. All other edges remain unchanged, i.e., if $(k, l) \in E \backslash(E(S(u), S(u)) \cup E(T(u), T(u)))$ then $(k, l) \in E^{\prime}$ and $w_{k l}=w_{k l}^{\prime}$. It is important to note that this construction does not change the degrees of the vertices in $V^{\prime} \backslash\left(S^{\prime}(u) \cup T^{\prime}(u)\right)$.

Consider the function $g: V^{\prime} \rightarrow \mathbb{R}$,

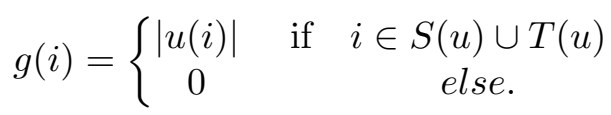

It can easily be checked that by construction of $\Gamma^{\prime}$, we have

$$
\begin{aligned}
\mu_{0} & =\frac{\sum_{e=(i, j) \in E} \mu_{i j}(u(i)+u(j))^{2}}{\sum_{i \in V} d_{i} u(i)^{2}} \\
& \geq \frac{\sum_{e^{\prime}=(i, j) \in E^{\prime}} w_{i j}^{\prime}(g(i)-g(j))^{2}}{\sum_{i \in V^{\prime}} d_{i}^{\prime} g(i)^{2}} \\
& \geq 1-\sqrt{1-\left(h^{\prime}(g)\right)^{2}}
\end{aligned}
$$

where we used Lemma 3.1 to obtain the last inequality. For any non-empty subset $W \subseteq S(g)=S(u) \cup T(u)$ we define $S_{1}=W \cap S(u)$ and $T_{1}=W \cap$ 
$T(u)$. Let $\emptyset \neq U \subseteq S(g)$ the subset that realizes the infimum, i.e.,

$$
\begin{aligned}
h^{\prime}(g) & =\inf _{\emptyset \neq W \subseteq S(g)} \frac{\left|E^{\prime}(W, \bar{W})\right|}{\operatorname{vol}(W)}=\frac{\left|E^{\prime}(U, \bar{U})\right|}{\operatorname{vol}(U)} \\
& =\frac{\left|E\left(S_{1}, S_{1}\right)\right|+\left|E\left(T_{1}, T_{1}\right)\right|+\left|E\left(S_{1} \cup T_{1}, \overline{S_{1} \cup T_{1}}\right)\right|}{\operatorname{vol}\left(S_{1}\right)+\operatorname{vol}\left(T_{1}\right)} \\
& =1-\frac{2\left|E\left(S_{1}, T_{1}\right)\right|}{\operatorname{vol}\left(S_{1}\right)+\operatorname{vol}\left(T_{1}\right)} \\
& \geq 1-\bar{h} .
\end{aligned}
$$

Thus, we have

$$
2-\lambda_{N-1}=\mu_{0} \geq 1-\sqrt{1-(1-\bar{h})^{2}}
$$

and so

$$
\lambda_{N-1} \leq 1+\sqrt{1-(1-\bar{h})^{2}}
$$

For example, the lower estimate for $\lambda_{N-1}$ in (3.10) is sharp if $\Gamma$ is a bipartite (by Theorem 3.1) or if $\Gamma$ is a complete graph $K_{N}$, and $N$ is even (by Example 4.1). In both examples, the partition that achieves $\bar{h}$ satisfies $V_{3}=\emptyset$. In fact, the proof of Theorem 3.2 shows that the estimate for $\lambda_{N-1}$ from below can only be sharp if $V_{3}=\emptyset$. However, if the volume of $V_{3}$ is sufficiently large, we can improve the estimate given in (3.10) and estimate the eigenvalue $\lambda_{N-1}$ from below by using both the Cheeger constant $h$ and its dual $\bar{h}$.

Corollary 3.1. Assume that $V_{1}, V_{2}$ and $V_{3}$ is a partition of $V$ that achieves $\bar{h}$. If

$$
\operatorname{vol}\left(V_{1} \cup V_{2}\right) \leq \operatorname{vol}\left(V_{3}\right)
$$

then

$$
\lambda_{N-1} \geq 2 \bar{h}+\mathcal{R}\left(V_{1}, V_{2}\right) h
$$

where we define for the partition $V_{1}, V_{2}, V_{3}$ of the vertex set $V$

$$
\mathcal{R}\left(V_{1}, V_{2}\right):=\frac{\min \left(\operatorname{vol}\left(V_{1}\right), \operatorname{vol}\left(V_{2}\right)\right)}{\max \left(\operatorname{vol}\left(V_{1}\right), \operatorname{vol}\left(V_{2}\right)\right)}
$$


Proof. The proof of Theorem 3.2 shows that

$$
\begin{aligned}
\lambda_{N-1} & \geq 2 \bar{h}+\mathcal{R}\left(V_{1}, V_{2}\right) \frac{\left|E\left(V_{1} \cup V_{2}, V_{3}\right)\right|}{\operatorname{vol}\left(V_{1}\right)+\operatorname{vol}\left(V_{2}\right)} \\
& =2 \bar{h}+\mathcal{R}\left(V_{1}, V_{2}\right) \frac{\left|E\left(V_{1} \cup V_{2}, V_{3}\right)\right|}{\min \left(\operatorname{vol}\left(V_{1} \cup V_{2}\right), \operatorname{vol}\left(V_{3}\right)\right)} \\
& \geq 2 \bar{h}+\mathcal{R}\left(V_{1}, V_{2}\right) h .
\end{aligned}
$$

The next corollary shows that if the eigenfunction for the largest eigenvalue $\lambda_{N-1}$ is sufficiently localized, then $\lambda_{N-1}$ can also be controlled from above in terms of the Cheeger constant $h$

Corollary 3.2. Let $u$ be the eigenfunction for the largest eigenvalue of $\Delta$. If the eigenfunction is sufficiently localized, i.e.,

$$
\sum_{i: u(i) \neq 0} d_{i} \leq \sum_{i: u(i)=0} d_{i}
$$

then

$$
\lambda_{N-1} \leq 1+\sqrt{1-h^{2}}
$$

Proof. Again we consider the smallest eigenvalue $\mu_{0}$ of the operator $L=I+P$ instead of the largest eigenvalue $\lambda_{N-1}$ of $\Delta$. Since $u$ is also an eigenfunction for the eigenvalue $\mu_{0}$ we have

$$
\begin{aligned}
\mu_{0} & =\frac{\sum_{e=(i, j)} \mu_{i j}(u(i)+u(j))^{2}}{\sum_{i} d_{i} u(i)^{2}} \\
& \geq \frac{\left.\sum_{e=(i, j)} w_{i j}|| u(i)|-| u(j)\right|^{2}}{\sum_{i} d_{i}|u(i)|^{2}} \\
& \geq 1-\sqrt{1-h^{2}(|u|)},
\end{aligned}
$$

where we used the reverse triangle inequality and Lemma 3.1. Since the eigenfunction is sufficiently localized, it follows from (3.13) that $\operatorname{vol}(S(u) \cup$ $T(u)) \leq \operatorname{vol}(\overline{S(u) \cup T(u)})$. This implies that $h(|u|)$ satisfies $h(|u|) \geq h$. Inserting this in the above equation yields

$$
2-\lambda_{n-1}=\mu_{0} \geq 1-\sqrt{1-h^{2}} .
$$


By considering a bipartite graph it becomes clear that Corollary 3.2 is in general not true if (3.13) is not satisfied. However, for infinite graphs the situation is different. For infinite graphs, the supremum of the spectrum can always be controlled from above in terms of a Cheeger constant, see Theorem 5.1. In Section 5, we develop a new approach that allows us to control the largest eigenvalue of a graph in terms of the Cheeger constant of its neighborhood graphs (instead of the Cheeger constant of the graph itself), see Corollary 5.1.

Proposition 3.2. Let $u$ be an eigenfunction for the largest eigenvalue of $\Delta$ that satisfies $\max _{i}|u(i)|=1$ then

$$
\lambda_{N-1} \leq 2-\frac{\min _{i, j} w_{i j}\left(1-\min _{i}|u(i)|\right)^{2}}{D \operatorname{vol}(V)},
$$

where $D$ is the diameter of the graph. In particular, if there exists a vertex $i$ such that $u(i)=0$ then

$$
\lambda_{N-1} \leq 2-\frac{\min _{i, j} w_{i j}}{D \operatorname{vol}(V)} .
$$

Proof. Again, we consider the smallest eigenvalue $\mu_{0}$ of $L=I+P$ instead of the largest eigenvalue $\lambda_{N-1}$ of $\Delta$. Let $i_{k}, k=1, \ldots, n$ be the shortest path connecting the vertices that satisfy $\max _{i}|u(i)|=\left|u\left(i_{1}\right)\right|=1$ and $\min _{i}|u(i)|=$ $\left|u\left(i_{n}\right)\right|$. Then we have

$$
\begin{aligned}
\mu_{0} & =\frac{\sum_{e=(i, j)} \mu_{i j}(u(i)+u(j))^{2}}{\sum_{i} d_{i} u(i)^{2}} \\
& \geq \frac{\left.\sum_{e=(i, j)} w_{i j}|| u(i)|-| u(j)\right|^{2}}{\sum_{i} d_{i}|u(i)|^{2}} \\
& \geq \min _{i, j} w_{i j} \frac{\sum_{k=1}^{n}\left(\left|u\left(i_{k}\right)\right|-\left|u\left(i_{k+1}\right)\right|\right)^{2}}{\operatorname{vol}(V)} .
\end{aligned}
$$

Using the Cauchy-Schwarz inequality we obtain

$$
\begin{aligned}
\mu_{0} & \geq \frac{\min _{i, j} w_{i j}}{n} \frac{\left(\sum_{k=1}^{n}\left|u\left(i_{k}\right)\right|-\left|u\left(i_{k+1}\right)\right|\right)^{2}}{\operatorname{vol}(V)} \\
& \geq \frac{\min _{i, j} w_{i j}}{D} \frac{\left(1-\min _{i}|u(i)|\right)^{2}}{\operatorname{vol}(V)}
\end{aligned}
$$

where we used the fact that the length of a shortest path connecting any two vertices is less or equal to $D$. Since $2-\lambda_{N-1}=\mu_{0}$, the proof is complete. 
In particular, the estimate in Proposition 3.2 is sharp for bipartite graphs, because then $|u(i)|=1$ for all $i \in V$.

Again, by using the concept developed in Section 5, we can derive a similar result to Proposition 3.2. In Corollary 5.2, we show that the largest eigenvalue can, independently of the corresponding eigenfunction, be controlled from above in terms of the diameter of the neighborhood graph.

Jerrum and Sinclair have shown how one can bound the Cheeger constant $h$ by using canonical paths $[31,32]$. Similarly, we can derive an upper bound for the dual Cheeger constant $\bar{h}$ by considering a suitable collection of paths. Let $\sigma_{i}$ be a path from vertex $i$ to vertex $i$ with an odd number of edges and let $\Sigma$ be the collection of all these paths (one for each vertex).

Theorem 3.3. We have

$$
\bar{h} \leq 1-\frac{1}{\xi}
$$

where

$$
\xi:=\max _{e=(k, l)} \frac{1}{w_{k l}} \sum_{i: \sigma_{i} \ni e=(k, l)} d_{i} .
$$

The sum is over all $i$ for which the path $\sigma_{i}$ contains the edge $e=(k, l)$.

Proof. For simplicity, we define the subset $\Omega \subset E$ as $\Omega:=E\left(V_{1}, V_{1}\right) \cup E\left(V_{1}\right.$, $\left.V_{3}\right) \cup E\left(V_{2}, V_{2}\right) \cup E\left(V_{2}, V_{3}\right)$. Now observe that for every vertex $i \in V_{1} \cup V_{2}$ a path $\sigma_{i}$ with an odd number of edges contains at least one edge in $\Omega$. Thus we have for any partition $V_{1}, V_{2}, V_{3}$ of the vertex set $V$

$$
\begin{aligned}
\operatorname{vol}\left(V_{1}\right)+\operatorname{vol}\left(V_{2}\right) & =\sum_{i \in V_{1} \cup V_{2}} d_{i} \\
& \leq \sum_{e=(k, l) \in \Omega} \sum_{i: \sigma_{i} \ni e=(k, l), i \in V_{1} \cup V_{2}} d_{i} \sum_{e=(k, l) \in \Omega} \frac{w_{k l}}{w_{k l}} d_{i} \\
& \leq \sum_{i=(k, l)} w_{k=(k, l) \in \Omega} \leq \xi|\Omega|
\end{aligned}
$$

Since this holds for all partitions, we have for the partition $V_{1}, V_{2}$ and $V_{3}$ that achieves $\bar{h}$

$$
1-\bar{h}=\frac{|\Omega|}{\operatorname{vol}\left(V_{1}\right)+\operatorname{vol}\left(V_{2}\right)} \geq \frac{1}{\xi} .
$$


Corollary 3.3. We have

$$
\xi \leq d_{\Gamma} w_{\Gamma} b_{\Gamma}
$$

where $d_{\Gamma}=\max _{i} d_{i}, \quad w_{\Gamma}=\frac{1}{\min _{i, j} w_{i j}} \quad$ and $\quad b_{\Gamma}=\max _{e} \#\{\sigma \in \Sigma: e \in \sigma\}$. Together with Theorems 3.2 and 3.3, this implies that

$$
\lambda_{N-1} \leq 1+\sqrt{1-\left(\frac{1}{d_{\Gamma} w_{\Gamma} b_{\Gamma}}\right)^{2}} .
$$

Remark. Diaconis and Stroock show in [16], by using a discrete analog of the Poincare inequality, that the largest eigenvalue satisfies

$$
\lambda_{N-1} \leq 2-\frac{2}{d_{\Gamma} w_{\Gamma} b_{\Gamma} \sigma_{\Gamma}}
$$

where $\sigma_{\Gamma}$ is the maximum number of edges in any $\sigma \in \Sigma$. A simple calculation shows that the estimate (3.14) obtained from the dual Cheeger inequality is better than the estimate (3.15) obtained from the Poincare inequality iff

$$
d_{\Gamma} w_{\Gamma} b_{\Gamma}<\frac{1}{\sigma_{\Gamma}}+\frac{\sigma_{\Gamma}}{4} .
$$

In general, it is not clear which of these estimates is better. See also the related discussion in [19], where the authors analyze when the Cheeger inequality improves the Poincare estimate for the smallest non-trivial eigenvalue.

\section{Relations between $h$ and $\bar{h}$}

By looking at the definitions of $h$ and $\bar{h}$ it is apparent that there is a connection between those two quantities. We shall explore this now in more detail.

Similarly to (3.12) we define:

Definition 4.1. For any partition $U, \bar{U}$ of the vertex set $V$ we define

$$
\mathcal{R}(U):=\frac{\min (\operatorname{vol}(U), \operatorname{vol}(\bar{U}))}{\max (\operatorname{vol}(U), \operatorname{vol}(\bar{U}))} .
$$

Furthermore,

$$
\mathcal{R}:=\max _{U} \mathcal{R}(U)
$$


First, we will restrict ourselves to unweighted graphs. Later on we will prove similar results for weighted graphs.

\section{Unweighted graphs:}

Lemma 4.1. Let $\Gamma$ be an unweighted graph with $N$ vertices, then

$$
\frac{N-1}{N+1} \leq \mathcal{R} \leq 1
$$

Equality holds on the left-hand side if and only if $\Gamma$ is a regular graph and $N$ is odd.

Proof. Order the vertices w.r.t. their degree, i.e., $d_{1} \geq d_{2} \geq \cdots \geq d_{N}$. We construct a partition $U, \bar{U}$ of $V$ that satisfies $\frac{N-1}{N+1} \leq \mathcal{R}(U)$. We begin with two empty sets $U_{0}, \bar{U}_{0}$. After the partition of $K$ vertices we denote the subsets by $U_{K}, \bar{U}_{K}$. Having started with vertex 1 as one of largest degree, we iteratively partition the vertices into two subsets such that vertex $K+1$ is then added to the subset $U_{K}, \bar{U}_{K}$ that has the smaller volume. We continue this procedure until we obtain a complete partition $U, \bar{U}:=U_{N}, \bar{U}_{N}$ of the vertex set $V$. Let $M \leq N$ be such that

$$
\operatorname{vol}\left(U_{M-1}\right) \geq \operatorname{vol}\left(\bar{U}_{M-1}\right)
$$

and

$$
\operatorname{vol}\left(\bar{U}_{K}\right) \geq \operatorname{vol}\left(U_{K}\right) \quad \text { for } M \leq K \leq N
$$

For simplicity, we define

$$
\operatorname{vol}\left(U_{K}\right)+\operatorname{vol}\left(\bar{U}_{K}\right)=: \operatorname{vol}\left(V_{K}\right) \text { for } 1 \leq K \leq N
$$

Then, we have

$$
\operatorname{vol}\left(\bar{U}_{M}\right)-\operatorname{vol}\left(U_{M}\right) \leq d_{M} \leq \frac{\operatorname{vol}\left(U_{M}\right)+\operatorname{vol}\left(\bar{U}_{M}\right)}{M}=\frac{\operatorname{vol}\left(V_{M}\right)}{M}
$$

Equality holds on the left-hand side if and only if $\operatorname{vol}\left(U_{M-1}\right)=\operatorname{vol}\left(\bar{U}_{M-1}\right)$ and equality holds on the right hand side if and only if $d_{1}=d_{2}=\cdots=d_{M}$. 
For the final partition $U_{N}, \bar{U}_{N}$ we obtain

$$
\begin{aligned}
\operatorname{vol}\left(\bar{U}_{N}\right)-\operatorname{vol}\left(U_{N}\right) & =\operatorname{vol}\left(\bar{U}_{M}\right)-\left(\operatorname{vol}\left(U_{M}\right)+\operatorname{vol}\left(V_{N}\right)-\operatorname{vol}\left(V_{M}\right)\right) \\
& \leq \frac{\operatorname{vol}\left(V_{M}\right)}{M}-\operatorname{vol}\left(V_{N}\right)+\operatorname{vol}\left(V_{M}\right) \\
& \leq \frac{\operatorname{vol}\left(V_{N}\right)}{N}
\end{aligned}
$$

The last inequality follows from:

$$
\begin{aligned}
& \frac{\operatorname{vol}\left(V_{N}\right)}{N}+\operatorname{vol}\left(V_{N}\right)-\operatorname{vol}\left(V_{M}\right)-\frac{\operatorname{vol}\left(V_{M}\right)}{M} \\
& \quad=\frac{1}{N M}\left[M(1+N) \operatorname{vol}\left(V_{N}\right)-N(1+M) \operatorname{vol}\left(V_{M}\right)\right] \\
& \quad=\frac{1}{N M}\left[M(1+N)\left(\operatorname{vol}\left(V_{N}\right)-\operatorname{vol}\left(V_{M}\right)\right)+(M-N) \operatorname{vol}\left(V_{M}\right)\right] \\
& \geq \frac{1}{N M}[M(1+N)(N-M)+(M-N) M N] \\
& \quad=\frac{(N-M)}{N} \geq 0
\end{aligned}
$$

Thus, we constructed a partition that satisfies

$$
\max \left(\operatorname{vol}\left(U_{N}\right), \operatorname{vol}\left(\bar{U}_{N}\right)\right)-\min \left(\operatorname{vol}\left(U_{N}\right), \operatorname{vol}\left(\bar{U}_{N}\right)\right) \leq \frac{\operatorname{vol}\left(V_{N}\right)}{N}
$$

Since

$$
\frac{\operatorname{vol}\left(V_{N}\right)}{N}=\frac{\max \left(\operatorname{vol}\left(U_{N}\right), \operatorname{vol}\left(\bar{U}_{N}\right)\right)+\min \left(\operatorname{vol}\left(U_{N}\right), \operatorname{vol}\left(\bar{U}_{N}\right)\right)}{N}
$$

this yields

$$
\frac{N-1}{N+1} \leq \mathcal{R}(U) \leq \mathcal{R}
$$

From the proof we see that equality holds iff $N=M$, the graph is regular, and $\operatorname{vol}\left(U_{M-1}\right)=\operatorname{vol}\left(\bar{U}_{M-1}\right)$. This implies that equality holds iff $\Gamma$ is regular and $N$ is odd.

The last lemma shows that for large graphs, i.e., $N$ large, it is always possible to partition $V$ into two subsets of almost equal volume. 
Corollary 4.1. In particular we have for unweighted graphs,

$$
\frac{1}{2} \leq \mathcal{R} \leq 1
$$

and equality holds on the left hand side iff $\Gamma$ is a triangle.

Proof. The proof follows from Lemma 4.1 since there exists only one connected graph on two vertices for which we have $\mathcal{R}=1$ and the only regular graph on three vertices is the triangle.

Theorem 4.1. For unweighted graphs we have

$$
\frac{N-1}{N} h \leq \frac{2 \mathcal{R}}{1+\mathcal{R}} h \leq \bar{h} \leq 1
$$

Equality holds on the l.h.s. iff $\Gamma$ is a regular graph and $N$ is odd.

Proof. By (3.5), we have for any partition $U, \bar{U}$ of the vertex set $V$

$$
h \leq \frac{|E(U, \bar{U})|}{\min (\operatorname{vol}(U), \operatorname{vol}(\bar{U}))}
$$

For the partition $V_{1}=U, V_{2}=\bar{U}$ and $V_{3}=\emptyset$ we obtain

$$
\bar{h} \geq \frac{2|E(U, \bar{U})|}{\operatorname{vol}(U)+\operatorname{vol}(\bar{U})} .
$$

Since this holds for all partitions $U, \bar{U}$, we get

$$
h \leq \bar{h} \frac{\operatorname{vol}(U)+\operatorname{vol}(\bar{U}))}{2 \min (\operatorname{vol}(U), \operatorname{vol}(\bar{U}))}=\bar{h}\left(\frac{1}{2}+\frac{1}{2 \mathcal{R}}\right) \leq \bar{h} \frac{N}{N-1}
$$

where we used Lemma 4.1. The remaining inequality follows from Theorem 3.1.

Corollary 4.2. Let $\Gamma$ be an unweighted graph. If there exists a partition $U, \bar{U}$ of the vertex set $V$ such that $\operatorname{vol}(U)=\operatorname{vol}(\bar{U})$ then

$$
h \leq \bar{h} \leq 1
$$

If $\Gamma=K_{2}$, we even have equality in (4.8), i.e., $h=\bar{h}=1$. Note that, in general there does not exist a partition $U, \bar{U}$ of $V$ such that $\operatorname{vol}(U)=\operatorname{vol}(\bar{U})$. 
Counterexamples are regular graphs if $N$ is odd or so-called wheel graphs $W_{N}$ with $N$ vertices and degree sequence $\pi=\{N-1,3, \ldots, 3\}$ if $N-1$ is not a multiple of 3 .

Example 4.1. For a complete graph $K_{N}$ on $N$ vertices, we have

$$
h= \begin{cases}\frac{N}{2(N-1)}, & N \text { even } \\ \frac{N+1}{2(N-1)}, & N \text { odd }\end{cases}
$$

and

$$
\bar{h}= \begin{cases}\frac{N}{2(N-1)}, & N \text { even } \\ \frac{N+1}{2 N}, & N \text { odd }\end{cases}
$$

This example shows that, for complete graphs, we have equality in (4.5) if $N$ is odd and in (4.8) if $N$ even, respectively.

\section{Weighted graphs:}

Lemma 4.1 does not hold for weighted graphs. This can be seen by considering sufficiently small weights $c$ in figure 1 . In particular, it turns out that

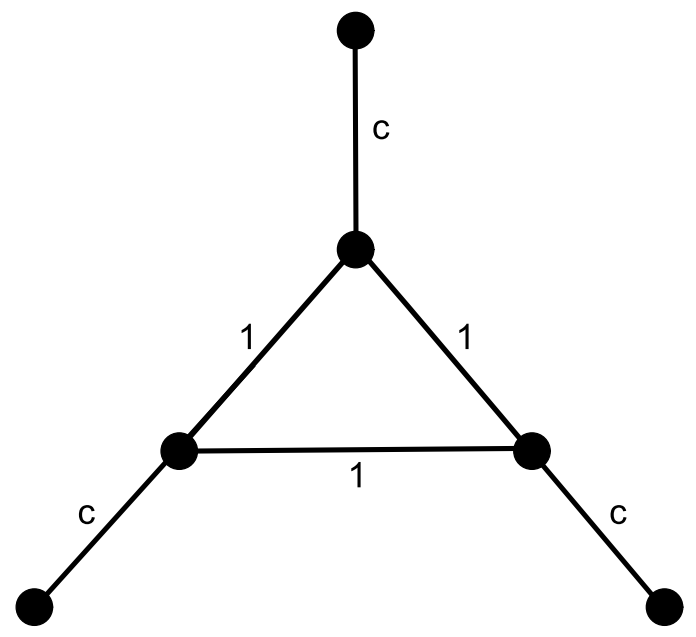

Figure 1: For sufficiently small weights $c$ this graph shows that Lemma 4.1 is not true for weighted graphs. 
inequality (4.4) does not hold for weighted graphs. However, we have the following result for weighted graphs.

Lemma 4.2. Let $\Gamma$ be a weighted graph with $N$ vertices and let $M \leq N$ be defined as in (4.2), then

$$
\frac{M-1}{M+1} \leq \mathcal{R} \leq 1
$$

Proof. Using the notation from the proof of Lemma 4.1 we conclude from (4.3):

$$
\begin{aligned}
\operatorname{vol}\left(\bar{U}_{N}\right)-\operatorname{vol}\left(U_{M}\right) & =\operatorname{vol}\left(\bar{U}_{M}\right)-\operatorname{vol}\left(U_{M}\right) \\
& \leq \frac{\operatorname{vol}\left(U_{M}\right)+\operatorname{vol}\left(\bar{U}_{M}\right)}{M} \\
& =\frac{\operatorname{vol}\left(U_{M}\right)+\operatorname{vol}\left(\bar{U}_{N}\right)}{M} .
\end{aligned}
$$

This implies

$$
\frac{M-1}{M+1} \leq \frac{\operatorname{vol}\left(U_{M}\right)}{\operatorname{vol}\left(\bar{U}_{N}\right)} \leq \frac{\operatorname{vol}\left(U_{N}\right)}{\operatorname{vol}\left(\bar{U}_{N}\right)} \leq \mathcal{R}
$$

Note that figure 1 does not contradict Lemma 4.2 for all $c>0$. Similarly to Theorem 4.1, we obtain for weighted graphs:

Theorem 4.2. Let $\Gamma$ be a weighted graph and let $M$ be defined as in (4.2), then

$$
\frac{M-1}{M} h \leq \frac{2 \mathcal{R}}{1+\mathcal{R}} h \leq \bar{h} \leq 1 .
$$

\section{Neighborhood graphs}

In the preceding sections, we have used geometric properties (like the Cheeger, the dual Cheeger constant or the diameter) of the underlying graph in order to control the eigenvalues of the graph Laplace operator.

In this section, we shall use a new, conceptually different approach in order to control the eigenvalues of the graph Laplace operator. Instead of using geometric properties of $\Gamma$ itself, we shall use the geometric properties of the neighborhood graph $\Gamma[l]$ of $\Gamma$, to be defined shortly, in order to control the eigenvalues of $\Delta$. 
As a motivation, consider the following result for infinite graphs [18, 23]:

Theorem 5.1. If $\Gamma$ is a locally finite graph, then

$$
1-\sqrt{1-\alpha^{2}(\Gamma)} \leq \inf \operatorname{spec}(\Delta) \leq \sup \operatorname{spec}(\Delta) \leq 1+\sqrt{1-\alpha^{2}(\Gamma)}
$$

where

$$
\alpha(\Gamma)=\inf _{W \subseteq V,|W|<\infty} \frac{E(W, \bar{W})}{\operatorname{vol}(W)}
$$

is a version of the Cheeger constant for an infinite graph.

Thus, for infinite graphs, it is possible to control the supremum and the infimum of $\operatorname{spec}(\Delta)$ by the Cheeger constant $\alpha(\Gamma)$. Clearly, this estimate is not useful for finite graphs as $\alpha(\Gamma)=0$ in that case, because we may then simply take $W=V$. The point here is that $\inf \operatorname{spec}(\Delta)=\lambda_{0}=0$ for finite graphs, but not necessarily for infinite graphs, and this is the content of the lower bound in Theorem 5.1 in qualitative terms. It is remarkable that the constant $\alpha(\Gamma)$ at the same time may also yield a non-trivial upper spectral bound for an infinite graph.

In the finite graph case we showed in Corollary 3.2 that a similar result to Theorem 5.1 is true if the eigenfunction that corresponds to the largest eigenvalue is sufficiently localized. In this section, we shall show (Corollary 5.1) that it is possible to control the maximal and the smallest nonzero eigenvalue of a finite graph in a similar way as in (5.1), if we use the Cheeger constant $h[l]$ of the neighborhood graph $\Gamma[l]$, for $l$ even, instead of the Cheeger constant $h$ of the graph $\Gamma$ itself. In particular, we obtain new lower bounds for the second smallest eigenvalue that can improve the classical Cheeger estimate (3.3); for a discussion and examples see the next section.

Definition 5.1. For a graph $\Gamma=(V, E)$ its neighborhood graph $\Gamma[l]=$ $(V, E[l])$ of order $l \geq 1$ is the graph with the same vertex set $V$ whose edge set $E[l]$ is defined in the following way: The weight $w_{i j}[l]$ of the edge $e[l]=(i, j)$ in $\Gamma[l]$ is given by

$$
w_{i j}[l]=\sum_{k_{1}, \ldots, k_{l-1}} \frac{1}{d_{k_{1}}} \cdots \frac{1}{d_{k_{l-1}}} w_{i k_{1}} w_{k_{1} k_{2}} \cdots w_{k_{l-1} j}
$$


if $l>1$ and we set $w_{i j}[l]=w_{i j}$ if $l=1$, i.e., $\Gamma[1]=\Gamma$. In particular, $i$ and $j$ are neighbors in $\Gamma[l]$ if there exists at least one path of length $l$ between $i$ and $j$ in $\Gamma$.

Remark. (i) The idea of neighborhood graphs is the following: define a family of graphs $\Gamma[l], l \geq 1$ that encodes the transition probabilities of the $l$-step random walk on the graph $\Gamma$.

We give an alternative definition of the neighborhood graphs. The neighborhood graph $\Gamma[l]=(V, E[l])$ of $\Gamma=(V, E)$ has the same vertex set $\mathrm{V}$ and the weights of the edges of $\Gamma[l]$ are defined by

$$
w_{i j}[l]:=P^{l}(i, j) d_{i}
$$

where $P^{l}(i, j)$ is the probability that a random walker starts at vertex $i$ and moves in $l$ steps to vertex $j$.

(ii) Neighborhood graphs are directly related to the discrete heat kernel $p_{t}(i, j)$ studied in [14]. We have the following relationship:

$$
p_{t}(i, j)=\frac{w_{i j}[t]}{d_{i} d_{j}}
$$

Lemma 5.1. The weights of the neighborhood graphs satisfy the following semi-group identity:

$$
w_{i j}[l]=\sum_{t} \frac{1}{d_{t}} w_{i t}[k] w_{t j}[l-k] \text { for } 1 \leq k<l .
$$

Proof. The proof follows from a direct calculation and we omit it here.

In order to become familiar with the concept of neighborhood graphs we consider the following examples:

Example 5.1. Consider the family of graphs in figure 2 for $c \geq 0$. Let $W$ be the adjacency operator of the graph in figure 2. $W$ can be represented as

$$
W=W[1]=\left(\begin{array}{ll}
c & 1 \\
1 & c
\end{array}\right) \text {. }
$$

If we go to higher order neighborhood graphs $\Gamma[l]$ of $\Gamma$, the topological structure remains the same and the adjacency operator $W[l], l=2,3,4,5$ 


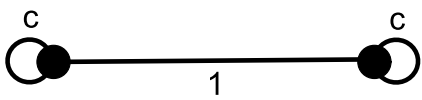

Figure 2: Graph in Example 5.1.

can be represented as

$$
\begin{aligned}
& W[2]=\left(\begin{array}{cc}
\frac{c^{2}+1}{1+c} & \frac{2 c}{1+c} \\
\frac{2 c}{1+c} & \frac{c^{2}+1}{1+c}
\end{array}\right) \\
& W[3]=\left(\begin{array}{cc}
\frac{c^{3}+3 c}{(1+c)^{2}} & \frac{3 c^{2}+1}{(1+c)^{2}} \\
\frac{3 c^{2}+1}{(1+c)^{2}} & \frac{c^{3}+3 c}{(1+c)^{2}}
\end{array}\right), \\
& W[4]=\left(\begin{array}{cc}
\frac{\left(c^{2}+1\right)^{2}+4 c^{2}}{(1+c)^{3}} & \frac{4 c^{3}+4 c}{(1+c)^{3}} \\
\frac{4 c^{3}+4 c}{(1+c)^{3}} & \frac{\left(c^{2}+1\right)^{2}+4 c^{2}}{(1+c)^{3}}
\end{array}\right) \\
& W[5]=\left(\begin{array}{cc}
\frac{c\left(5+10 c^{2}+c^{4}\right)}{(1+c)^{4}} & \frac{1+10 c^{2}+5 c^{4}}{(1+c)^{4}} \\
\frac{1+10 c^{2}+5 c^{4}}{(1+c)^{4}} & \frac{c\left(5+10 c^{2}+c^{4}\right)}{(1+c)^{4}}
\end{array}\right) .
\end{aligned}
$$

Example 5.2. As a second example we consider the family of graphs in figure 3. We have

$$
\begin{gathered}
W=W[1]=\left(\begin{array}{llllll}
0 & c & c & 0 & 0 & 0 \\
c & 0 & c & 0 & 0 & 0 \\
c & c & 0 & 1 & 0 & 0 \\
0 & 0 & 1 & 0 & c & c \\
0 & 0 & 0 & c & 0 & c \\
0 & 0 & 0 & c & c & 0
\end{array}\right) \text { and } \\
W[2]=\left(\begin{array}{cccccc}
c / 2+c^{2} / a & c^{2} / a & c / 2 & c / a & 0 & 0 \\
c^{2} / a & c / 2+c^{2} / a & c / 2 & c / a & 0 & 0 \\
c / 2 & c / 2 & 1 / a+c & 0 & c / a & c / a \\
c / a & c / a & 0 & 1 / a+c & c / 2 & c / 2 \\
0 & 0 & c / a & c / 2 & c / 2+c^{2} / a & c^{2} / a \\
0 & 0 & c / a & c / 2 & c^{2} / a & c / 2+c^{2} / a
\end{array}\right),
\end{gathered}
$$

where $a:=1+2 c$. 


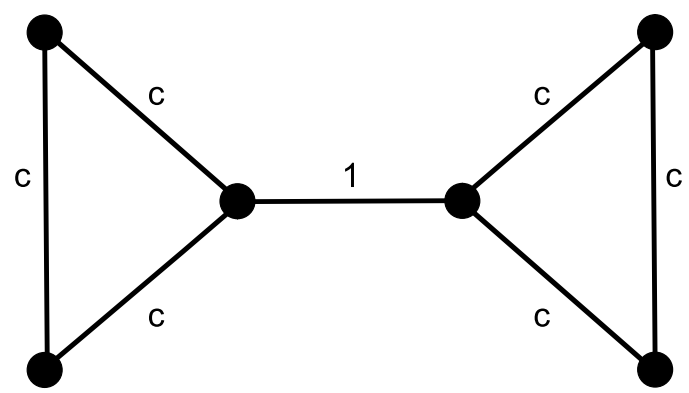

Figure 3: Graph in Example 5.2.

The neighborhood graphs $\Gamma[l]$ have the following property:

Lemma 5.2. $d_{i}=d_{i}[l]$ for all $i \in V$ and $l \geq 1$.

Proof. We have

$$
\begin{aligned}
d_{i}[l] & =\sum_{j} w_{i j}[l]=\sum_{k_{1}, \ldots, k_{l-1}} \frac{1}{d_{k_{1}}} \cdots \frac{1}{d_{k_{l-1}}} w_{i k_{1}} w_{k_{1} k_{2}} \cdots w_{k_{l-2} k_{l-1}} \sum_{j} w_{k_{l-1} j} \\
& =\sum_{k_{1}, \ldots, k_{l-2}} \frac{1}{d_{k_{1}}} \cdots \frac{1}{d_{k_{l-2}}} w_{i k_{1}} w_{k_{1} k_{2}} \cdots \sum_{k_{l-1}} w_{k_{l-2} k_{l-1}} \\
& \vdots \\
& =\sum_{k_{1}} w_{i k_{1}}=d_{i} .
\end{aligned}
$$

Remark. Lemma 5.2 implies that $(\cdot, \cdot)_{\mu}=(\cdot, \cdot)_{\mu[l]}$ and thus $\ell^{2}(V, \mu)=$ $\ell^{2}(V, \mu[l])$, as will be frequently utilized below.

Theorem 5.2. For any function $u \in \ell^{2}(V, \mu)$ we have

$$
\left(I-(I-\Delta)^{l}\right) u=\left(I-P^{l}\right) u=\Delta[l] u
$$

where $P$ is the transition probability operator of a random walk on $\Gamma$ and $\Delta[l]$ is the graph Laplace operator on $\Gamma[l]$. 
Proof. For any function $u \in \ell^{2}(V, \mu)$, we have

$$
\left(I-P^{l}\right) u(i)=u(i)-\sum_{j} q_{i j} u(j)
$$

where $q_{i j}:=\sum_{k_{1}, \ldots, k_{l-1}} \frac{w_{i k_{1}}}{d_{i}} \cdots \frac{w_{k_{l-1} j}}{d_{k_{l-1}}}$ is $i j$-th entry of $P^{l}$. Thus,

$$
\left(I-P^{l}\right) u(i)=u(i)-\sum_{j} \sum_{k_{1}, \ldots, k_{l-1}} \frac{w_{i k_{1}}}{d_{i}} \cdots \frac{w_{k_{l-1} j}}{d_{k_{l-1}}} u(j) .
$$

Using the definition $w_{i j}[l]=\sum_{k_{1}, \ldots, k_{l-1}} \frac{1}{d_{k_{1}}} \cdots \frac{1}{d_{k_{l-1}}} w_{i k_{1}} w_{k_{1} k_{2}} \cdots w_{k_{l-1} j}$ and $d_{i}=d_{i}[l]$, this yields

$$
\begin{aligned}
\left(I-P^{l}\right) u(i) & =\frac{1}{d_{i}[l]} \sum_{j} w_{i j}[l](u(i)-u(j)) \\
& =\Delta[l] u(i) .
\end{aligned}
$$

Lemma 5.3. Let $\Gamma$ be a graph and $\Gamma[l]$ its neighborhood graph of order $l$.

(i) If $\Gamma$ is connected and $l$ is even, then $\Gamma[l]$ consists of exactly two connected components iff $\Gamma$ is bipartite.

(ii) If $l$ is odd, then $\Gamma[l]$ is bipartite iff $\Gamma$ is bipartite. Furthermore, $\Gamma[l]$ has the same number of connected components as $\Gamma$.

(iii) The multiplicity $m_{1}$ of the eigenvalue one is an invariant for all neighborhood graphs, i.e., $m_{1}(\Delta)=m_{1}(\Delta[l])$ for all $l \geq 1$.

(iv) The eigenvalues of $\Delta[l]$ satisfy

$$
0=\lambda_{0}[l] \leq \cdots \lambda_{N-1}[l] \leq 1
$$

if $l$ is even and

$$
0=\lambda_{0}[l] \leq \cdots \lambda_{N-1}[l] \leq 2
$$

if $l$ is odd.

(v) If $\lambda \neq 0,2$ then $\lambda[l]=1-(1-\lambda)^{l} \rightarrow 1$ as $l \rightarrow \infty$.

Proof. $\Gamma$ and $\Gamma[l]$ have the same vertex set, thus both $\Delta$ and $\Delta[l]=(I-$ $\left.(I-\Delta)^{l}\right)$ have $N=|V|$ eigenvalues. Furthermore, every eigenfunction $u_{k}$ 
for $\Delta$ and eigenvalue $\lambda_{k}$ is also an eigenfunction for $\Delta[l]$ and eigenvalue $1-\left(1-\lambda_{k}\right)^{l}$.

(i) If $l$ is even, $\lambda_{k}[l]=0$ iff $\lambda_{k}=0$ or $\lambda_{k}=2$. Recall that the multiplicity of the eigenvalue zero is equal to the number of connected components of a graph and 2 is an eigenvalue iff the graph is bipartite. Now let $\Gamma$ be a connected, bipartite graph and let $l$ be even. Then $\lambda[l]=0$ is twice in the spectrum of $\Delta[l]$ since $\lambda_{0}=0$ and $\lambda_{N-1}=2$ are in the spectrum of $\Delta$. Consequently, $\Gamma[l]$ consists of exactly two connected components. On the other hand if $\Gamma[l]$ consists of exactly two connected components, $\lambda[l]=0$ is twice in the spectrum of $\Delta[l]$, and we know that either the eigenvalue $\lambda=0$ is twice in the spectrum of $\Delta$ or $\lambda=0$ and $\lambda=2$ are both in the spectrum of $\Delta$. Since we assume that $\Gamma$ is connected, $\lambda=0$ is a simple eigenvalue, and thus we can conclude that $2 \in \operatorname{spec}(\Delta)$ and $\Gamma$ is bipartite.

(ii) - (v) follow from simple calculations.

Remark. By Lemma 5.3 (iv) all eigenvalues of $\Delta[l]$ ( $l$ even) are less or equal to 1 . From (2.22) we observe that this is only possible because $\Gamma[l]$ ( $l$ even) contains many (in fact $N$ ) loops. In contrast, for a graphs without loops we have $1<\frac{N}{N-1} \leq \lambda_{N-1}$.

We now use Theorem 5.2 to control the eigenvalues of $\Delta$ on $\Gamma$ in terms of geometric properties of its neighborhood graphs $\Gamma[l]$.

Theorem 5.3. Let $\mathcal{A}[l]$ be a lower bound for the eigenvalue $\lambda_{1}[l]$ of $\Delta[l]$, i.e., $\mathcal{A}[l] \leq \lambda_{1}[l]$. Then, the eigenvalues of $\Delta$ satisfy

$$
1-(1-\mathcal{A}[l])^{\frac{1}{l}} \leq \lambda_{1} \leq \cdots \leq \lambda_{N-1} \leq 1+(1-\mathcal{A}[l])^{\frac{1}{l}},
$$

if $l$ is even and

$$
1-(1-\mathcal{A}[l])^{\frac{1}{l}} \leq \lambda_{1}
$$

if $l$ is odd. 
Proof. Let $u_{k}, k \neq 0$ be an eigenfunction for $\Delta$. Using (5.3) we obtain

$$
\begin{aligned}
1-\left(1-\lambda_{k}\right)^{l} & =\frac{\left(u_{k},\left(I-(I-\Delta)^{l}\right) u_{k}\right)_{\mu}}{\left(u_{k}, u_{k}\right)_{\mu}} \\
& =\frac{\left(u_{k}, \Delta[l] u_{k}\right)_{\mu[l]}}{\left(u_{k}, u_{k}\right)_{\mu[l]}} \\
& \geq \inf _{u:(u, \mathbf{1})_{\mu[l]}=0} \frac{(u, \Delta[l] u)_{\mu[l]}}{(u, u)_{\mu[l]}} \\
& =\lambda_{1}[l] \geq \mathcal{A}[l],
\end{aligned}
$$

since $(u, \mathbf{1})_{\mu}=0$ iff $(u, \mathbf{1})_{\mu[l]}=0$. Alternatively, since the eigenvalues of $\Delta[l]$ are given by $1-\left(1-\lambda_{k}\right)^{l}$ if $\lambda_{k}$ are the eigenvalues of $\Delta$ we have

$$
1-\left(1-\lambda_{k}\right)^{l} \geq \min _{k \neq 0} 1-\left(1-\lambda_{k}\right)^{l}=\lambda_{1}[l] \geq \mathcal{A}[l] .
$$

This implies that for all $k \neq 0$

$$
\left|1-\lambda_{k}\right| \leq(1-\mathcal{A}[l])^{\frac{1}{l}}
$$

if $l$ is even and

$$
1-\lambda_{k} \leq(1-\mathcal{A}[l])^{\frac{1}{l}}
$$

if $l$ is odd.

As a concrete example, we use the Cheeger inequality (3.3) as a lower bound for $\lambda_{1}[l]$, i.e., $\mathcal{A}[l]=1-\sqrt{1-h^{2}[l]} \leq \lambda_{1}[l]$, where $h[l]$ is the Cheeger constant of the neighborhood graph $\Gamma[l]$.

Corollary 5.1. The eigenvalues of $\Delta$ on $\Gamma$ satisfy:

$$
1-\left(1-h^{2}[l]\right)^{\frac{1}{2 l}} \leq \lambda_{1} \leq \cdots \leq \lambda_{N-1} \leq 1+\left(1-h^{2}[l]\right)^{\frac{1}{2 l}}
$$

if $l$ is even and

$$
1-\left(1-h^{2}[l]\right)^{\frac{1}{2 l}} \leq \lambda_{1}
$$

if $l$ is odd.

We point out that this result is similar to Theorem 5.1 for locally finite graphs. However, the difference is that we have to use here the Cheeger constant of the neighborhood graph instead of the Cheeger constant itself. 
As another example, we use the well-known estimate $\lambda_{1} \geq \frac{\min _{i, j} w_{i j}}{D \operatorname{vol}(V)}$ for the smallest non-trivial eigenvalue in terms of the diameter and the volume of a graph; see for instance [10]. This yields the following estimates:

Corollary 5.2. All eigenvalues of $\Delta$ satisfy

$$
1-\left(1-\frac{\min _{i, j} w_{i j}[l]}{D[l] \operatorname{vol}(V[l])}\right)^{\frac{1}{l}} \leq \lambda_{1} \leq \cdots \leq \lambda_{N-1} \leq 1+\left(1-\frac{\min _{i, j} w_{i j}[l]}{D[l] \operatorname{vol}(V[l])}\right)^{\frac{1}{l}}
$$

if $l$ is even and

$$
1-\left(1-\frac{\min _{i, j} w_{i j}[l]}{D[l] \operatorname{vol}(V[l])}\right)^{\frac{1}{l}} \leq \lambda_{1}
$$

if $l$ is odd.

Remark. Some graph properties, like the discrepancy or the expansion property of a graph, can be controlled by the quantity $\rho=\max _{k \neq 0}\left|1-\lambda_{k}\right|$ [10]. Corollary 5.1 and Corollary 5.2 or more generally Theorem 5.3 can be used to derive explicit bounds for those quantities. As one particular application, we show in Section 9 how Corollary 5.1 can be used to control the convergence of random walks on graphs.

We can use Theorem 5.2 to obtain further eigenvalue estimates.

Theorem 5.4. Let $\mathcal{B}[l]$ be any upper bound for $\lambda_{1}[l]$, i.e., $\lambda_{1}[l] \leq \mathcal{B}[l]$. Then the eigenvalues of $\Delta$ satisfy

$$
\lambda_{1} \leq 1-(1-\mathcal{B}[l])^{\frac{1}{l}}
$$

or

$$
\lambda_{N-1} \geq 1+(1-\mathcal{B}[l])^{\frac{1}{l}}
$$

if $l$ is even and

$$
\lambda_{1} \leq 1-(1-\mathcal{B}[l])^{\frac{1}{l}}
$$

if $l$ is odd.

Proof. First note that, by Lemma 5.3, (5.8) and (5.9) are well defined, if $l$ is even, since we can assume w.l.o.g. that $\mathcal{B}[l] \leq 1$. Using (5.3), we obtain $\mathcal{B}[l] \geq$ 
$\lambda_{1}[l]=\min _{k \neq 0} 1-\left(1-\lambda_{k}\right)^{l}$. This implies that for at least one eigenvalue $\lambda_{i}$, $i \neq 0$ we have

$$
(1-\mathcal{B}[l])^{\frac{1}{l}} \leq\left|1-\lambda_{i}\right|
$$

if $l$ is even and

$$
(1-\mathcal{B}[l])^{\frac{1}{l}} \leq 1-\lambda_{i}
$$

if $l$ is odd.

Using the Cheeger inequality (3.2) for $\Gamma[l]$ we obtain:

Corollary 5.3. If $l$ is even, and $2 h[l] \leq 1$, then we have

$$
\lambda_{1} \leq 1-(1-2 h[l])^{\frac{1}{l}}
$$

or

$$
\lambda_{N-1} \geq 1+(1-2 h[l])^{\frac{1}{l}}
$$

If $l$ is odd, we have

$$
\lambda_{1} \leq 1-(1-2 h[l])^{\frac{1}{l}}
$$

In the next section, we show that the estimate (5.10) and (5.12) for $\lambda_{1}$ can improve the Cheeger estimate (3.2).

Theorem 5.5. Let $\mathcal{C}[l]$ be any lower bound for the largest eigenvalue $\lambda_{N-1}[l]$, i.e., $\mathcal{C}[l] \leq \lambda_{N-1}[l]$. At least one eigenvalue of $\Delta$ is contained in the interval

$$
\left[1-(1-\mathcal{C}[l])^{\frac{1}{l}}, 1+(1-\mathcal{C}[l])^{\frac{1}{l}}\right]
$$

if $l$ is even and the largest eigenvalue of $\Delta$ satisfies

$$
\lambda_{N-1} \geq 1-(1-\mathcal{C}[l])^{\frac{1}{l}}
$$

if $l$ is odd.

Proof. Again, by Lemma 5.3, (5.13) is well defined, if $l$ is even, since $\mathcal{C}[l] \leq$ $\lambda_{N-1}[l] \leq 1$. Using $(5.3)$ we have $\mathcal{C}[l] \leq \lambda_{N-1}[l]=\max _{k}\left(1-\left(1-\lambda_{k}\right)^{l}\right)$. 
Thus,

$$
\min _{k}\left|1-\lambda_{k}\right| \leq(1-\mathcal{C}[l])^{\frac{1}{l}}
$$

if $l$ is even and

$$
\min _{k}\left(1-\lambda_{k}\right)=1-\max _{k} \lambda_{k} \leq(1-\mathcal{C}[l])^{\frac{1}{l}}
$$

if $l$ is odd.

In particular, we have from Theorem 3.2 and Theorem 5.5:

Corollary 5.4. If $l$ is even, and $2 \bar{h}[l] \leq 1$, then at least one eigenvalue of $\Delta$ is contained in the interval

$$
\left[1-(1-2 \bar{h}[l])^{\frac{1}{l}}, 1+(1-2 \bar{h}[l])^{\frac{1}{l}}\right] .
$$

If $l$ is odd, then the largest eigenvalue satisfies

$$
\lambda_{N-1} \geq 1-(1-2 \bar{h}[l])^{\frac{1}{l}} .
$$

We now turn to the gap phenomenon for eigenvalues, that is, find some interval that does not contain any eigenvalue.

Theorem 5.6. Let $\mathcal{D}[l]$ be any upper bound for the largest eigenvalue, i.e., $\lambda_{N-1}[l] \leq \mathcal{D}[l]$. Then all eigenvalues of $\Delta$ are contained in the union of intervals

$$
\left[0,1-(1-\mathcal{D}[l])^{\frac{1}{l}}\right] \bigcup\left[1+(1-\mathcal{D}[l])^{\frac{1}{l}}, 2\right]
$$

if $l$ is even and the largest eigenvalue of $\Delta$ satisfies

$$
\lambda_{N-1} \leq 1-(1-\mathcal{D}[l])^{\frac{1}{l}}
$$

if $l$ is odd.

The proof is similar to the proofs above so we omit it here. We only note that (5.15) is well defined if $l$ is even since, by Lemma 5.3, we can assume w.l.o.g. that $\mathcal{D}[l] \leq 1$.

In other words: let $l$ be even, then for any upper bound $\mathcal{D}[l] \leq 1$ none of the eigenvalues of $\Delta$ is contained in the interval $\left(1-(1-\mathcal{D}[l])^{\frac{1}{l}}, 1+(1-\right.$ $\left.\mathcal{D}[l])^{\frac{1}{l}}\right)$. Thus, if $l$ is even, an upper bound $\mathcal{D}[l] \leq 1$ for $\lambda_{N-1}[l]$ of $\Delta[l]$ can be used to bound all eigenvalues of $\Delta$ on $\Gamma$ away from 1 . In particular, if 
$\mathcal{D}[l]<1, \Delta$ then does not possess the eigenvalue 1 ; see also Lemma 5.3. In [3], it was observed that the eigenvalue 1 occurs in an unweighted graph whenever there are two nodes $i_{1}, i_{2}$ that are not neighbors themselves, but who possess the same neighbors, that is, for any $k$, we have $k \sim i_{1}$ iff $k \sim i_{2}$. Thus, a graph satisfying the assumptions of Theorem 5.6 cannot have any such pair of nodes if $\mathcal{D}[l]<1$ and $l$ is even.

From the dual Cheeger inequality (3.10), we only obtain an upper bound for $\lambda_{N-1}$ if $l$ is odd since $\mathcal{D}[l]=1+\sqrt{1-(1-\bar{h}[l])^{2}} \geq 1$ for all $l$.

Corollary 5.5. If $l$ is odd, then

$$
\lambda_{N-1} \leq 1+\left(1-(1-\bar{h}[l])^{2}\right)^{\frac{1}{2 l}} .
$$

\section{Comparison of the Cheeger estimates with the estimates obtained by the neighborhood graph method}

In the following, we compare the Cheeger estimates (3.2) and (3.3) with the new estimates in Corollary 5.1 and Corollary 5.3. Recall that Corollary 5.1 and Corollary 5.3 were obtained by applying (3.2) and (3.3) to the neighborhood graph $\Gamma[l]$ and then using the relationship between the spectrum of $\Delta$ and $\Delta[l]$, which was established in Theorem 5.2.

Comparing Corollary 5.1 with (3.3) reveals that our new estimates improve the Cheeger estimate (3.3) if

$$
h[l] \geq \sqrt{1-\left(1-h^{2}\right)^{l}},
$$

for some $l \geq 2$. In general, it is not clear for which graphs $\Gamma$ and which $l$ the equation (6.1) is satisfied. However, we can develop some qualitative intuition about (6.1).

We have to distinguish whether $l$ is even or odd. Assume for the moment that $l$ is even. Clearly, (6.1) is not satisfied whenever $\Gamma$ is bipartite since then, by Lemma $5.2, \Gamma[l]$ is disconnected and so $h[l]=0$. In fact, Corollary 5.1 yields only the trivial estimate $0 \leq \lambda_{1}$ for bipartite graphs. In contrast, for graphs that are not bipartite the estimate in (5.6) always yields a non-trivial lower bound $0<1-\left(1-h^{2}[l]\right)^{\frac{1}{2 l}} \leq \lambda_{1}$ for the second smallest eigenvalue $\lambda_{1}$. A necessary condition for strict inequality in (6.1) is that $h[l]>h$. In order to understand for which graphs it is likely that this necessary condition is satisfied we distinguish the following two cases. If $\lambda_{1} \geq 2-\lambda_{N-1}$ then $\lambda_{1}[l]=1-\left(1-\lambda_{N-1}\right)^{l}$. In this case it is possible that $\lambda_{1}[l]<\lambda_{1}$. Hence, in general, we cannot expect that $h[l]>h$ is satisfied, unless the Cheeger 
estimate on the graph $\Gamma[l]$ is sharper (this will be made more precise in the next proposition) than the Cheeger estimate on the graph $\Gamma$. In particular, if $\lambda_{1} \geq 2-\lambda_{N-1}$, we cannot expect that (6.1) is satisfied. On the other hand, if $\lambda_{1}<2-\lambda_{N-1}$ then $\lambda_{1}[l]=1-\left(1-\lambda_{1}\right)^{l}>\lambda_{1}$ and so it is likely that the necessary condition $h[l]>h$ is satisfied. Roughly speaking, if $l$ is even, we can expect that our eigenvalue estimates improve the Cheeger estimates if the graph in question is closer to disconnected graph than to bipartite graph.

If $l$ is odd then Corollary 5.1 always yields non-trivial estimates and $\lambda_{1}[l]>\lambda_{1}$ is always satisfied. Thus, we can expect that the necessary condition $h[l]>h$ is, in general, satisfied if $l$ is odd.

After all, we are mainly interested in the question when the estimates in Corollary 5.1 improve the Cheeger estimate (3.3)

Proposition 6.1. Let $S[l]:=\frac{1-\sqrt{1-h^{2}[l]}}{\lambda_{1}[l]}$ be the sharpness of the Cheeger estimate (3.3) on the graph $\Gamma[l]$, i.e., the closer $S[l]$ is to 1 the sharper is the Cheeger estimate. We set $S[1]=: S$ and $h[1]=: h$. If one of the following two conditions is satisfied

(i) $l$ is odd and $S[l]$ satisfies

$$
S[l] \geq \frac{1-\left(1-h^{2}\right)^{\frac{l}{2}}}{1-\left[1-\frac{1}{S}\left(1-\sqrt{1-h^{2}}\right)\right]^{l}},
$$

(ii) $l$ is even, $S[l]$ satisfies (6.2), and $\lambda_{1} \leq \lambda_{N-1}$,

then the estimates in Corollary 5.1 improve the Cheeger estimate (3.3).

Proof. We have

$$
\begin{aligned}
S[l] & =\frac{1-\sqrt{1-h^{2}[l]}}{\lambda_{1}[l]}=\frac{1-\sqrt{1-h^{2}[l]}}{1-\left(1-\lambda_{1}\right)^{l}} \\
& =\frac{1-\sqrt{1-h^{2}[l]}}{1-\left[1-\frac{1}{S}\left(1-\sqrt{1-h^{2}}\right)\right]^{l}} .
\end{aligned}
$$

A comparison with (6.2) yields

$$
1-\sqrt{1-h^{2}[l]} \geq 1-\left(1-h^{2}\right)^{\frac{l}{2}}
$$

which implies (6.1).

As an example we consider again the family of graphs in Example 5.1. The corresponding Cheeger constants of the neighborhood graphs are given 


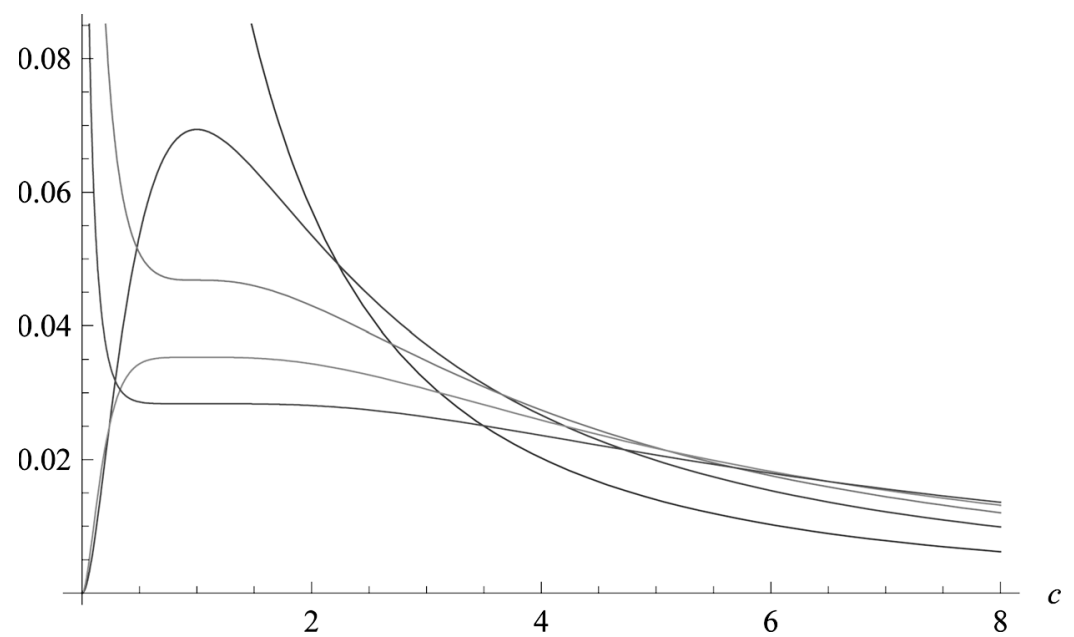

Figure 4: Plot of different lower bounds $1-\left(1-h^{2}[l]\right)^{\frac{1}{2 l}}$ for the second smallest eigenvalue $\lambda_{1}$ of the family of graphs in figure 2 . The Cheeger estimate $(l=1)$ is plotted in dark blue, and the red, yellow, green and light blue curves correspond to $l=2,3,4$, and $l=5$, respectively.

by $\quad h[1]=\frac{1}{1+c}, \quad h[2]=\frac{2 c}{(1+c)^{2}}, \quad h[3]=\frac{3 c^{2}+1}{(1+c)^{3}}, \quad h[4]=\frac{4 c^{3}+4 c}{(1+c)^{4}}, \quad$ and $\quad h[5]=$ $\frac{1+10 c^{2}+c^{4}}{(1+c)^{5}}$. In figure 4 , we plot the lower bounds $1-\left(1-h[l]^{2}\right)^{\frac{1}{2 l}}$, for the second smallest eigenvalue $\lambda_{1}$, for different values of $l$. We observe that for $c<2.2$ the Cheeger inequality $(l=1)$ yields the best estimate for the second smallest eigenvalue $\lambda_{1}$ of the graph in figure 2. However, if we increase $c$ the estimates for larger values of $l$ become better. This confirms the intuition that graphs that are closer to disconnected than to bipartite graphs are likely to satisfy (6.1).

As a second example, we consider the family of graphs in Example 5.2. The Cheeger constants of the neighborhood graphs are given by $h[1]=$ $\min \left\{\frac{1}{6 c+1}, \frac{1}{2}\right\}, \quad h[2]=\min \left\{\frac{4 c}{(6 c+1)(2 c+1)}, \frac{5 c+8 c^{2}}{2\left(1+6 c+8 c^{2}\right)}\right\}, \quad$ and $\quad h[3]=\min$ $\left\{\frac{12 c^{2}+4 c+1}{(1+2 c)^{2}(1+6 c)}, \frac{c\left(12 c^{2}+12 c+7\right)}{(1+2 c)^{2} \min \{8 c, 2+4 c\}}\right\}$. In figure 5 , the lower bounds $1-(1-$ $\left.h[l]^{2}\right)^{\frac{1}{2 l}}$, for the second smallest eigenvalue $\lambda_{1}$, are plotted for $l=1,2,3$. Again, for small $c$ the Cheeger estimate, $l=1$ yields the best estimate. However, if $c>0.8$ then the estimate for $l=3$ improves the Cheeger estimate.

We can also compare the upper bound for $\lambda_{1}$ in Corollary 5.3 with the Cheeger inequality (3.2). We observe that if $l$ is odd and

$$
h[l] \leq \frac{1-(1-2 h)^{l}}{2}
$$




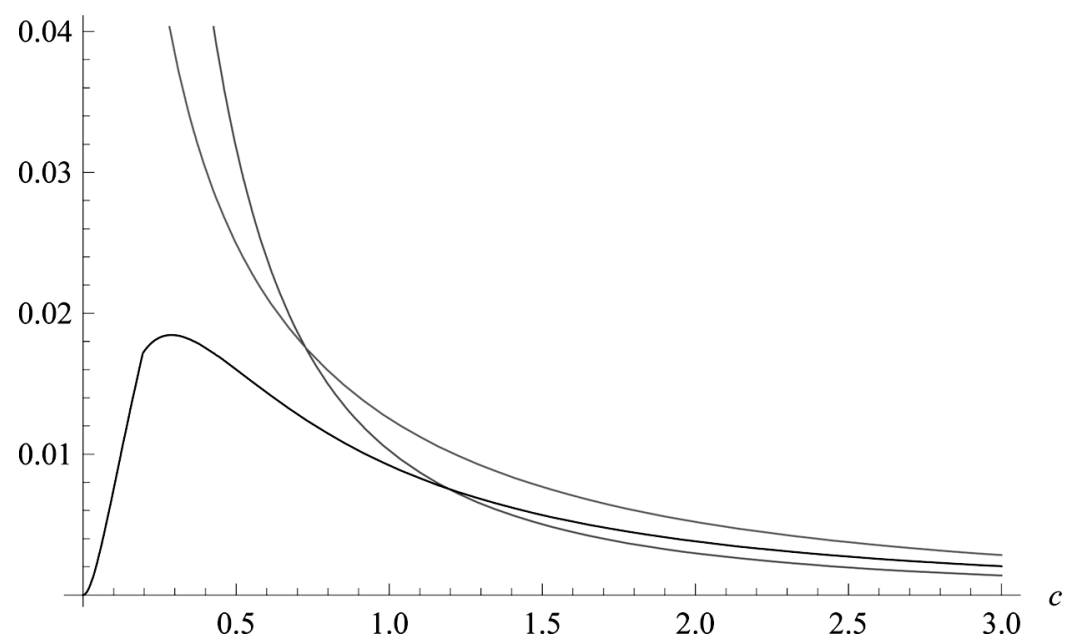

Figure 5: Plot of different lower bounds $1-\left(1-h^{2}[l]\right)^{\frac{1}{2 l}}$, for the second smallest eigenvalue of the family of graphs in figure 3 . The Cheeger estimate $(l=1)$ is plotted in blue, and the black, red curves correspond to $l=2$, and $l=3$, respectively.

is satisfied then the estimates in Corollary 5.3 improve the Cheeger estimate (3.2). If $l$ is even, $h[l] \leq \frac{1}{2}$, (6.3) is satisfied, and $\lambda_{1} \leq 2-\lambda_{N-1}$, then Corollary 5.3 improves the Cheeger estimate (3.2). If $l$ is even, we have to assume that $\lambda_{1} \leq 2-\lambda_{N-1}$, because otherwise (5.11) holds instead of (5.10) and so we do not always have an upper bound for $\lambda_{1}$. Similarly to Proposition 6.1 we obtain:

Proposition 6.2. Let $s[l]:=\frac{\lambda_{1}[l]}{2 h[l]}$ be the sharpness of the upper Cheeger estimate for the second smallest eigenvalue $\lambda_{1}[l]$ of $\Gamma[l]$. If one of the following two conditions is satisfied

(i) $l$ is odd and

$$
s[l] \geq \frac{1-(1-s 2 h)^{l}}{1-(1-2 h)^{l}} .
$$

(ii) $l$ is even and in addition to (6.4), $h[l] \leq \frac{1}{2}$, and $\lambda_{1} \leq 2-\lambda_{N-1}$, then the estimates in Corollary 5.3 improve the Cheeger estimate (3.2).

The proof is straightforward so we omit it here. 


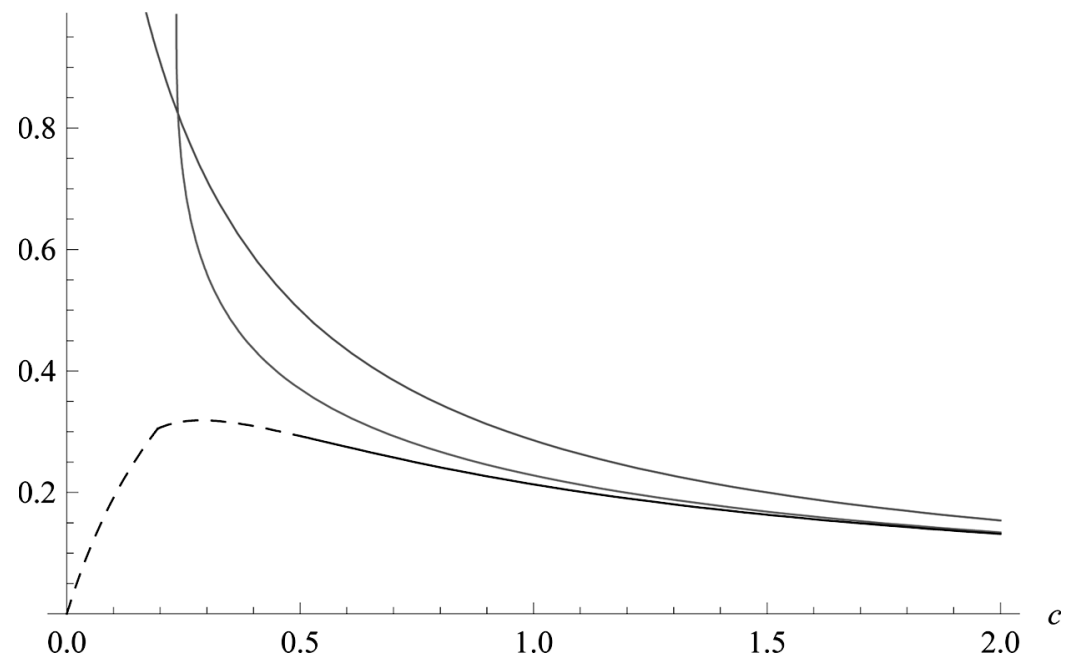

Figure 6: Plot of different upper bounds $1-(1-2 h[l])^{\frac{1}{l}}$, for the second smallest eigenvalue of the family of graphs in figure 3 . The Cheeger estimate $(l=1)$ is plotted in blue, and the black, red curves correspond to $l=2$, and $l=3$, respectively. The dashed black line indicates that for $c \leq 0.5$ this is not an upper bound for $\lambda_{1}$ because in this case (5.11) holds and (5.10) is not satisfied.

It turns out that, if we consider the family of graphs in Example 5.1, the different upper bounds $\lambda_{1} \leq 1-(1-2 h[l])^{\frac{1}{l}}$ for the second smallest eigenvalue are the same for all $l$. In contrast for the family of graphs in Example 5.2 the plot in figure 6 shows that the estimate in Corollary 5.3 can improve the Cheeger estimate (3.2). For example, if $c>0.3$ the estimate for $l=3$ improves the Cheeger estimate (3.2). Comparing figures 5 and 6 show that the estimates in Corollaries 5.1 and 5.3 improve both Cheeger estimates (3.2) and (3.3) at the same time if $c>0.8$ and $l=3$.

Finally, we observe that if $h[l]$ is not contained in the interval $\left[\frac{1-(1-2 h)^{l}}{2}, \sqrt{1-\left(1-h^{2}\right)^{l}}\right]$ (where the interval is the empty set if $\frac{1-(1-2 h)^{l}}{2}>$ $\left.\sqrt{1-\left(1-h^{2}\right)^{l}}\right)$ then at least one of the Cheeger estimates (3.2) and (3.3) is improved by the estimates in Corollaries 5.1 and 5.3.

Similarly, one can also compare the dual Cheeger estimate for the largest eigenvalue in Theorem 3.2 with the estimates obtained in Corollary 5.5. We observe that, similarly to the Cheeger estimate, the neighborhood graph method can improve the estimates obtained from the dual Cheeger estimate. As one such example we consider the family of graphs in Example 5.1. In 


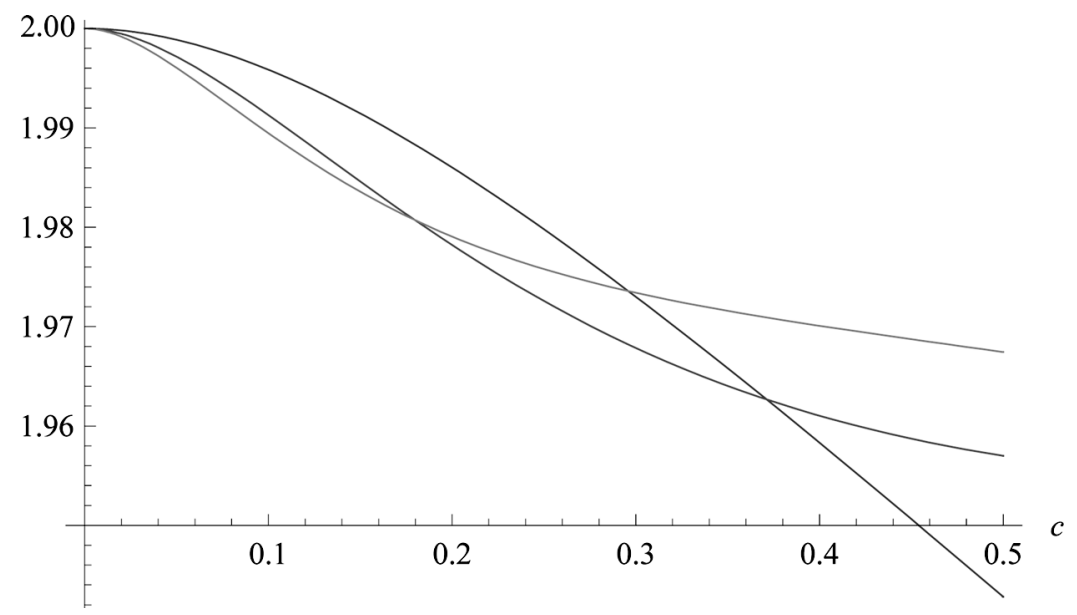

Figure 7: Plot of different upper bounds $1+\left(1-(1-\bar{h}[l])^{2}\right)^{\frac{1}{2 l}}$ in Corollary 5.5 for the largest eigenvalue of the family of graphs in figure 2 . The dual Cheeger estimate (Theorem 3.2) $(l=1)$ is plotted in dark blue, and the red and yellow curves correspond to $l=3$ and $l=5$, respectively. Note that for the family of graphs in figure 2 we have $\bar{h}[l]=h[l]$.

figure 7 , we plot different upper bounds $1+\left(1-(1-\bar{h}[l])^{2}\right)^{\frac{1}{2 l}}$ for the largest eigenvalue $\lambda_{N-1}$. For the largest eigenvalue, one can derive similar results as in (6.1) and Proposition 6.1. However, we do not want to go into further detail here because the calculations are exactly the same as before.

We conclude this section by noting that the neighborhood graph method is very powerful because it may improve any known eigenvalue estimate.

\section{An example}

As discussed above, the highest eigenvalue $\lambda_{N-1}$ of $\Delta$ becomes largest for bipartite and smallest for complete graphs, respectively. And a guiding question for this paper is what can we say about the highest eigenvalue of graphs that are neither bipartite nor complete, i.e., what structural properties of $\Gamma$ lead to a highest eigenvalue $\lambda_{N-1}$ close to 2 , or very different from 2 , respectively.

In order to develop some further intuition about the highest eigenvalue, we now consider the following example. Let $\Gamma_{0}$ be a bipartite graph with $N$ vertices. We consider a highest eigenfunction $\bar{u}$ that is +1 on one class and 
-1 on the other class of vertices, as described above. In particular by (2.17),

$$
\frac{\frac{1}{2} \sum_{k} \sum_{j} w_{k j}(\bar{u}(j)-\bar{u}(k))^{2}}{\sum_{i} d_{i} \bar{u}(i)^{2}}=2 .
$$

By adding another vertex $i_{0}$ and connecting it to one of the vertices $i_{1}$ of $\Gamma_{0}$ we obtain a new bipartite graph $\Gamma_{1}$. We extend $\bar{u}$ by $\bar{u}\left(i_{0}\right)=0$ to $\Gamma_{1}$. Thus, the numerator and the denominator of (7.1) are both increased by $w_{i_{0} i_{1}}$. Let $\Gamma_{0}$ be sufficiently large, i.e., $\sum_{i} d_{i}$ is sufficiently large, then we can achieve for $\Gamma_{1}$ that for any given small $\epsilon>0$,

$$
\frac{\frac{1}{2} \sum_{k} \sum_{j} w_{k j}(\bar{u}(j)-\bar{u}(k))^{2}}{\sum_{i} d_{i} \bar{u}(i)^{2}}>2-\epsilon .
$$

Now, this is not affected when we construct a graph $\Gamma$ by attaching another graph $\Gamma_{2}$ at $i_{0}$ and extend $\bar{u}$ by 0 to all of $\Gamma_{2}$. For instance, $\Gamma_{2}$ could be a complete graph $K_{M}$ with $M$ vertices, for any $M$. In particular, the difference $2-\lambda_{N-1}$ which has to be smaller than $\epsilon$ by (2.16), is not very sensitive to the shape of $\Gamma_{2}$. This implies, for instance, that $2-\lambda_{N-1}$ cannot reflect a global quantity like the clustering coefficient $C$ of (2.1) that expresses an averaged difference from a graph being bipartite. In fact, our construction of attaching a complete graph $K_{M}$ to a bipartite graph $\Gamma_{0}$ through a connecting node produces a graph with $C$ arbitrarily close to its maximal value 1 when $M$ is sufficiently large.

By extending this example, we can also see that we should have many eigenvalues $\lambda$ for which $2-\lambda$ is small when the graph possesses several relatively large bipartite or almost bipartite parts that are only loosely connected with the rest. (By (5.3), the neighborhood graph $\Gamma[2]$ of such a graph contains several large components that are only loosely connected, i.e., many eigenvalues $\lambda_{k}[2]$ that are small.) This is analogous to the fact that a graph possesses several small eigenvalues when it has many relatively large components that are only loosely connected to the rest, that is, when the graph can be easily decomposed into several large clusters. Of course, for a non-connected graph, that is, one with several components without links between them, the spectrum simply is the union of the spectra of the components. Therefore, by the continuity principle, a graph consisting of clusters that are only loosely connected to each other has its spectrum approximated by the spectra of these clusters, that is, 
by the one of the graph resulting from deleting the few links between the clusters.

\section{Controlling the largest eigenvalue in terms of a local clustering coefficient}

In this section, we shall provide a more technical estimate from above for the highest eigenvalue $\lambda_{N-1}$. For that purpose, we shall first derive some general identity, for a function $u$ on the vertex set $V$ of $\Gamma$.

Lemma 8.1. Let $u$ be an eigenfunction of $\Delta$ for the eigenvalue $\lambda$. Then, the following identity holds:

$$
2-\lambda=\frac{(\Delta[2] u, u)_{\mu}}{(\Delta u, u)_{\mu}}=\frac{\sum_{i} \frac{1}{d_{i}} \sum_{j, k} w_{i j} w_{i k}(u(j)-u(k))^{2}}{\sum_{i} \sum_{j} w_{i j}(u(i)-u(j))^{2}} .
$$

Remark. This identity follows from Theorem 5.2 for $l=2$ and (2.12) applied to the neighborhood graph. Here, as an alternative, we shall provide a direct proof.

Proof.

$$
\begin{aligned}
& \sum_{i} \frac{1}{d_{i}} \sum_{j, k} w_{i j} w_{i k}(u(j)-u(k))^{2} \\
& \quad=\sum_{i} \frac{1}{d_{i}}\left(\sum_{j, k} w_{i k} w_{i j} u(j)^{2}-2 \sum_{j, k} w_{i j} w_{i k} u(j) u(k)+\sum_{j, k} w_{i j} w_{i k} u(k)^{2}\right) \\
& \quad=2 \sum_{i}\left(\sum_{j} w_{i j} u(j)^{2}-\frac{1}{d_{i}}\left(\sum_{j} w_{i j} u(j)\right)^{2}\right) \\
& \quad=2 \sum_{i} \sum_{j} w_{i j} u(j)^{2}-\sum_{i} 2 d_{i}\left(\frac{1}{d_{i}} \sum_{j} w_{i j} u(j)\right)^{2} .
\end{aligned}
$$


We now observe that we can replace $u$ by $u-u(i)$ in the first and hence also in all subsequent lines. This yields

$$
\begin{aligned}
& \sum_{i} \frac{1}{d_{i}} \sum_{j, k} w_{i j} w_{i k}(u(j)-u(k))^{2} \\
& \quad=2 \sum_{i} \sum_{j} w_{i j}(u(j)-u(i))^{2}-\sum_{i} 2 d_{i}\left(\frac{1}{d_{i}} \sum_{j} w_{i j}(u(j)-u(i))\right)^{2} \\
& \quad=2 \sum_{i} \sum_{j} w_{i j}(u(j)-u(i))^{2}-\sum_{i} 2 d_{i}(\Delta u(i))^{2}
\end{aligned}
$$

Since $u$ is an eigenfunction, $\Delta u=\lambda u$ for some eigenvalue $\lambda$, then, recalling (2.17), we obtain

$$
\sum_{i} \frac{1}{d_{i}} \sum_{j, k} w_{i j} w_{i k}(u(j)-u(k))^{2}=2 \lambda(2-\lambda) \sum_{i} d_{i} u(i)^{2} .
$$

Using (2.17) again, we can also reformulate this as

$$
2-\lambda=\frac{\sum_{i} \frac{1}{d_{i}} \sum_{j, k} w_{i j} w_{i k}(u(j)-u(k))^{2}}{\sum_{i} \sum_{j} w_{i j}(u(i)-u(j))^{2}} .
$$

We also observe, by a reasoning similar to the one for Lemma 8.1:

Lemma 8.2. Let $u$ be an eigenfunction of $\Delta$ for the eigenvalue $\lambda$. Then,

$$
2-\lambda=\frac{2 \sum_{i} \sum_{k} w_{i k}\left(\frac{1}{d_{i}} \sum_{j} w_{i j}(u(j)-u(k))\right)^{2}}{\sum_{i} \sum_{j} w_{i j}(u(j)-u(i))^{2}} .
$$

We now employ (8.1) to interpret $2-\lambda_{N-1}$ as quantifying how much $\Gamma$ is locally different from being bipartite. Recall that this quantity is 0 iff $\Gamma$ happens to be bipartite. Note that (8.3) can also be used to estimate the local difference from being bipartite in terms of $2-\lambda_{N-1}$.

As discussed above, the (global) clustering coefficient $C$ is not an appropriate measure for the difference $2-\lambda_{N-1}$. For instance, in Section 7 we constructed a graph whose largest eigenvalue is close to 2 although its clustering coefficient is close to 1 . However, we shall see that it is possible to 
control $2-\lambda_{N-1}$ by the following local clustering measure

$$
C_{0}:=\min _{e=(i, j)} \frac{\alpha_{i}+\alpha_{j}}{2}
$$

where

$$
\alpha_{i}:=\frac{\sum_{j: e=(i, j) \in \triangle} w_{i j}}{d_{i}}
$$

and $e=(i, k) \in \triangle(i \in \triangle)$ denotes that the edge $e=(i, k)$ (the vertex $i$ ) is contained in some triangle. Hence, $\alpha_{i}$ is the fraction of weights $w_{i j}$ for fixed $i$ that are contained in some triangle. In particular, if $i \notin \triangle$ then $\alpha_{i}=0$. Again, $C_{0}=0$ for a bipartite and $C_{0}=1$ for a complete graph. Furthermore, we define

$$
W:=\left(\min _{i \in \triangle k: e=(i, k) \in \triangle} \sum_{l:(i, k, l) \in \triangle} \frac{d_{i}}{d_{l}} \frac{w_{l i} w_{l k}}{w_{i k}}\right)^{1 / 2}
$$

and

$$
\bar{d}:=\max _{i \in \triangle} d_{i}
$$

Theorem 8.1. The largest eigenvalue $\lambda_{N-1}$ of $\Delta$ can be controlled from above by

$$
2-\frac{1}{2 \bar{d}} C_{0}\left(\frac{W}{1+W}\right)^{2}=: 2-H \geq \lambda_{N-1} .
$$

Proof. First we rewrite (8.1) for the largest eigenvalue $\lambda_{N-1}$ in the following form:

$$
\begin{gathered}
\sum_{e=(i, j)}\left(\frac{1}{d_{i}} \sum_{k} \mu_{i j} w_{i k}\left(u_{N-1}(j)-u_{N-1}(k)\right)^{2}\right. \\
\left.+\frac{1}{d_{j}} \sum_{k} \mu_{j i} w_{j k}\left(u_{N-1}(i)-u_{N-1}(k)\right)^{2}\right) \\
2-\lambda_{N-1}=\frac{\left.u_{N-1}(j)\right)^{2}+\mu_{j i}\left(u_{N-1}(j)-u_{N-1}(i)\right)^{2}}{\sum_{e=(i, j)} \mu_{i j}\left(u_{N-1}(i)-u_{N}\right.}
\end{gathered}
$$

where again $\mu_{i j}=w_{i j}$ if $i \neq j$ and $\mu_{i j}=\frac{1}{2} w_{i j}$ if $i=j$. In order to control $2-\lambda_{N-1}$ from below we need to match any term $\mu_{i j}\left(u_{N-1}(i)-u_{N-1}(j)\right)^{2}$ in the denominator by some term in the numerator of comparable magnitude. Since there is nothing to match if $i=j$ we can use the weights $w_{i j}$ instead of 
$\mu_{i j}$ throughout the proof. For ease of notation we will drop the index $N-1$ in the rest of the proof.

For simplicity, we first match the term $w_{i j}(u(i)-u(j))^{2}$ in the denominator with $\frac{1}{d_{i}} \sum_{k} w_{i j} w_{i k}(u(j)-u(k))^{2}$ in the numerator. Because of the symmetry in $i$ and $j$ the second term in the numerator and denominator can be treated in the same way.

Let $K_{1}(i) \subset V$ be the set of all neighbors $k$ of $i$ for which $e=(i, k) \in \triangle$ and

$$
(u(j)-u(k))^{2} \geq \gamma_{i j}^{2}(u(i)-u(j))^{2}
$$

is satisfied. The constant $\gamma_{i j}$ will be used later on to minimize our upper bound for $\lambda_{N-1}$. Similarly, let $K_{2}(i) \subset V$ be the set of all neighbors $k$ of $i$ which satisfy $e=(i, k) \in \triangle$ and

$$
(u(j)-u(k))^{2}<\gamma_{i j}^{2}(u(i)-u(j))^{2} .
$$

Clearly,

$$
\sum_{k \in K_{1}(i) \cup K_{2}(i)} w_{i k}=\sum_{k: e=(i, k) \in \triangle} w_{i k}=\alpha_{i} d_{i}
$$

If $i \notin \triangle$ then $\alpha_{i}=0$ and $K_{1}(i)=K_{2}(i)=\emptyset$. We distinguish the following two cases:

( $i$ ) Assume that $\sum_{k \in K_{1}(i)} w_{i k} \geq \frac{\alpha_{i} d_{i}}{2}$ is satisfied for vertex $i$. Consequently, there exists a term

$$
\frac{1}{d_{i}} \sum_{k} w_{i j} w_{i k}(u(j)-u(k))^{2} \geq \frac{\alpha_{i}}{2} \gamma_{i j}^{2} w_{i j}(u(i)-u(j))^{2}
$$

in the numerator. Thus, the term $w_{i j}(u(i)-u(j))^{2}$ in the numerator is matched.

(ii) Now assume that $\sum_{k \in K_{2}(i)} w_{i k} \geq \frac{\alpha_{i} d_{i}}{2}$ is satisfied for vertex $i$. We can not directly match $w_{i j}(u(i)-u(j))^{2}$ by using (8.10) because it could happen that $\frac{1}{d_{i}} \sum_{k} w_{i j} w_{i k}(u(j)-u(k))^{2}=0$. However, Equation (8.10) can be used to find a term of comparable size in the numerator. (8.10) implies that all neighbors $k$ of $i$ in $K_{2}(i)$ satisfy

$$
(u(i)-u(k))^{2}>\left(1-\gamma_{i j}\right)^{2}(u(i)-u(j))^{2} .
$$


Now the idea is to use the terms in the numerator several times in order to match $w_{i j}(u(i)-u(j))^{2}$. Multiplying the numerator in (8.1) by $w_{i j}$ yields

$$
\begin{aligned}
& w_{i j} \sum_{l} \frac{1}{d_{l}} \sum_{m, k} w_{l m} w_{l k}(u(m)-u(k))^{2} \\
& =w_{i j} \sum_{l} \frac{1}{d_{l}} \sum_{k} w_{l i} w_{l k}(u(i)-u(k))^{2} \\
& \quad+w_{i j} \sum_{l} \frac{1}{d_{l}} \sum_{k, m \neq i} w_{l m} w_{l k}(u(m)-u(k))^{2} .
\end{aligned}
$$

Now, we will only use the first term on the r.h.s. The second term can be used to match other terms in the denominator. The first term on the r.h.s. of (8.13) yields:

$$
\begin{aligned}
& w_{i j} \sum_{l} \frac{1}{d_{l}} \sum_{k} w_{l i} w_{l k}(u(i)-u(k))^{2} \\
& \geq w_{i j} \sum_{l} \frac{1}{d_{l}} \sum_{k \in K_{2}(i)} w_{l i} w_{l k}(u(i)-u(k))^{2} \\
& =w_{i j} \sum_{k \in K_{2}(i)} \sum_{l:(i, k, l) \in \triangle} \frac{1}{d_{l}} w_{l i} w_{l k}(u(i)-u(k))^{2} \\
& >\left(1-\gamma_{i j}\right)^{2} w_{i j}(u(i)-u(j))^{2}\left(\sum_{k \in K_{2}(i)}^{\sum_{i k}}\right)_{k \in K_{2}(i)} \sum_{l:(i, k, l) \in \triangle} \frac{1}{d_{l}} \frac{w_{l i} w_{l k}}{w_{i k}} \\
& \quad \geq\left(1-\gamma_{i j}\right)^{2} w_{i j}(u(i)-u(j))^{2} \frac{\alpha_{i}}{2} \underbrace{\min _{l:(i, k, l) \in \triangle} \frac{d_{i}}{d_{l}} \frac{w_{l i} w_{l k}}{w_{i k}}}_{k: e=(i, k) \in \triangle} .
\end{aligned}
$$

Thus, $w_{i j}(u(i)-u(j))^{2}$ is matched in the numerator. In order to match all other terms of the form $w_{i p}(u(i)-u(p))^{2}$ in the denominator for fixed $i$ we need to use the terms in the numerator at most $\sum_{p} w_{i p}=d_{i}$ times. Note that we can use the second term on the r.h.s. of (8.13) in order to match other terms of the form $w_{m p}(u(m)-u(p))^{2}$ for $m \neq i$. If some vertex $q$ is not contained in a triangle we have $\alpha_{q}=0$ and thus we do not need to match the terms $w_{q p}(u(q)-u(p))^{2}$ for fixed $q$. We conclude that we used the terms in the numerator at most $\max _{i \in \triangle} d_{i}$ times. Owing to the symmetry in $i$ and 
$j$ the second term in the numerator (8.8) can be treated in the same way. We obtain the following estimate:

$$
2-\lambda_{N-1} \geq \frac{1}{2 \bar{d}} \min _{e=(i, j)} \max _{\gamma_{i j}} \min \left\{a_{i j}, b_{i j}, c_{i j}, d_{i j}\right\}
$$

where

$$
\begin{aligned}
a_{i j} & :=\frac{\alpha_{i}+\alpha_{j}}{2} \gamma_{i j}^{2}, \\
b_{i j} & :=\frac{\alpha_{i}}{2} \gamma_{i j}^{2}+\frac{\alpha_{j}}{2} W^{2}\left(1-\gamma_{i j}\right)^{2}, \\
c_{i j} & :=\frac{\alpha_{i}}{2} W^{2}\left(1-\gamma_{i j}\right)^{2}+\frac{\alpha_{j}}{2} \gamma_{i j}^{2}, \\
d_{i j} & :=\frac{\alpha_{i}}{2} W^{2}\left(1-\gamma_{i j}\right)^{2}+\frac{\alpha_{j}}{2} W^{2}\left(1-\gamma_{i j}\right)^{2},
\end{aligned}
$$

and $W^{2}:=\min _{i \in \triangle} A(i)$.

Choosing $\gamma_{i j}=\frac{W}{1+W}$ yields

$$
2-\lambda_{N-1} \geq \frac{1}{2 \bar{d}} C_{0}\left(\frac{W}{1+W}\right)^{2}
$$

Remark. Similarly as the local clustering coefficient, the Olliver-Ricci curvature on a graph [28] is related to the relative abundance of triangles. In [6] it is shown that the largest eigenvalue of $\Delta$ can be controlled from above by a lower bound for the Olliver-Ricci curvature on graphs.

With the scheme developed in this section, the control in the other direction, that is, estimating the largest eigenvalue from below, does not quite work, because of the following example. Consider a graph with many cycles of odd length, but all of them of length at least 5 . Here, $C_{0}(\Gamma)=0$ as there are no triangles, but $2-\lambda_{N-1} \neq 0$ because the graph is not bipartite as bipartite graphs can only have cycles of even length.

However, we can control the largest eigenvalue from below in a different way, as we have seen in Section 3. 


\section{Random walks on graphs and the convergence to equilibrium}

We have seen that neighborhood graphs are deeply related to random walks on graphs. Hence, it is not surprising that the techniques developed in Sections 3 and 5 can be applied to random walks on graphs. We recall the following theorem for the convergence of random walks on graphs [20].

Theorem 9.1. For any function $f \in \ell^{2}(V, \mu)$, set

$$
\bar{f}=\frac{1}{\operatorname{vol}(V)} \sum_{j} d_{j} f(j) .
$$

Then for any positive integer $t$, we have

$$
\left\|P^{t} f-\bar{f}\right\| \leq \rho^{t}\|f\|
$$

where $\rho=\max _{k \neq 0}\left|1-\lambda_{k}\right|=\max \left\{\left|1-\lambda_{1}\right|,\left|1-\lambda_{N-1}\right|\right\}$ is the spectral radius of the transition probability operator of a random walk $P$ and $\|f\|=$ $\sqrt{(f, f)_{\mu}}$. Consequently, if $\Gamma$ is connected and not bipartite, then

$$
\left\|P^{t} f-\bar{f}\right\| \rightarrow 0
$$

as $t \rightarrow \infty$, i.e., $P^{t} f$ converges to a constant $\bar{f}$ as $t \rightarrow \infty$.

We define the equilibrium transition probability operator $\bar{P}: \ell^{2}(V, \mu) \rightarrow$ $\ell^{2}(V, \mu)$ as

$$
\bar{P} u(i)=\frac{1}{\operatorname{vol}(V)} \sum_{j} d_{j} u(j) .
$$

For all functions $f \in \ell^{2}(V, \mu)$ we have, $\bar{P} f=\bar{f}$ and thus, by (9.1), $P^{t}$ converges to $\bar{P}$ as $t \rightarrow \infty$ (if $\Gamma$ is not bipartite). As expected, the equilibrium transition probability for going from $i$ to $j$ only depends on the degree of vertex $j$ (and the volume of the graph, i.e., the sum of all degrees). In addition, we define the equilibrium weighted adjacency operator as $\bar{W}:=D \bar{P}$, where $D: \ell^{2}(V, \mu) \rightarrow \ell^{2}(V, \mu)$ is the multiplication operator defined as

$$
D u(i)=d_{i} u(i)
$$

The adjacency operator of $\Gamma[l]$ is given by $W[l]=D P^{l}$. Hence, if $\Gamma$ is not bipartite, $\Gamma[l]$ converges to the equilibrium graph $\bar{\Gamma}$ as $l \rightarrow \infty$ (in the sense 
that $W[l]$ converges to $\bar{W})$. The equilibrium graphs of the families of graphs studied in Examples 5.1 and 5.2 can be represented as

$$
\bar{W}=\left(\begin{array}{cc}
\frac{1+c}{2} & \frac{1+c}{2} \\
\frac{1+c}{2} & \frac{1+c}{2}
\end{array}\right)
$$

and

$$
\bar{W}=\left(\begin{array}{cccccc}
a_{1} & a_{1} & a_{2} & a_{2} & a_{1} & a_{1} \\
a_{1} & a_{1} & a_{2} & a_{2} & a_{1} & a_{1} \\
a_{2} & a_{2} & a_{3} & a_{3} & a_{2} & a_{2} \\
a_{2} & a_{2} & a_{3} & a_{3} & a_{2} & a_{2} \\
a_{1} & a_{1} & a_{2} & a_{2} & a_{1} & a_{1} \\
a_{1} & a_{1} & a_{2} & a_{2} & a_{1} & a_{1}
\end{array}\right)
$$

where $a_{1}=\frac{4 c^{2}}{12 c+2}, a_{2}=\frac{4 c^{2}+2 c}{12 c+2}$, and $a_{3}=\frac{4 c^{2}+4 c+1}{12 c+2}$.

If $\Gamma$ is bipartite then $\Gamma[l]$ does not converge as $l \rightarrow \infty$. This can for example be seen from Lemma 5.2. For a bipartite graph $\Gamma, \Gamma[l]$ is disconnected and not bipartite whenever $l$ is even and $\Gamma[l]$ is connected and bipartite whenever $l$ is odd. However, for a bipartite graph $\Gamma$, the subsequence of neighborhoods graphs $\Gamma[l]$ for $l$ even converges to $\bar{\Gamma}_{l \text { even }}$ as $l \rightarrow \infty$. The corresponding operator $\bar{W}_{l \text { even }}$ can be represented as

$$
\bar{W}_{l \text { even }}=\left(\begin{array}{cc}
\bar{W}_{1} & 0 \\
0 & \bar{W}_{2}
\end{array}\right),
$$

where $\left(\bar{W}_{k}\right)_{i j}=\frac{2 d_{i} d_{j}}{\operatorname{vol}(V)}=\frac{d_{i} d_{j}}{\operatorname{vol}\left(V_{k}\right)}$, for $k=1,2$, if $i$ and $j$ belong to the same subset $V_{k}$ and $V_{1}, V_{2}$ yields a bipartite decomposition of the vertex set $V$. Thus, $\bar{\Gamma}_{l}$ even is the disjoint union of two complete graphs of size $\left|V_{1}\right|$ and $\left|V_{2}\right|$. Similarly, if $\Gamma$ is bipartite, the subsequence $\Gamma[l]$ for $l$ odd converges to $\bar{\Gamma}_{l \text { odd }}$ as $l \rightarrow \infty$. In this case, the corresponding operator $\bar{W}_{l \text { odd }}$ can be represented as

$$
\bar{W}_{l \text { odd }}=\left(\begin{array}{cc}
0 & \bar{W}_{1} \\
\bar{W}_{2} & 0
\end{array}\right),
$$

where $\left(\bar{W}_{k}\right)_{i j}=\frac{2 d_{i} d_{j}}{\operatorname{vol}(V)}$, for $k=1,2$, if $i$ and $j$ belong to different subsets. Thus, $\bar{\Gamma}_{l}$ odd is the complete bipartite graph that has same bipartite decomposition $V_{1}, V_{2}$ of vertex set $V$ as $\Gamma$.

From Theorem 9.1 we see that we need to control the spectral radius $\rho$ of the transition probability operator $P$. Our results in Sections 3 and 5 
allow us to control $\rho$ in various different ways. For example, Corollary 5.1 implies that

$$
\rho=\max _{k \neq 0}\left|1-\lambda_{k}\right| \leq\left(1-h^{2}[l]\right)^{\frac{1}{2 l}}, \text { for all } l \text { even }
$$

This gives us the following explicit estimates for Theorem 9.1:

\section{Theorem 9.2.}

$$
\left\|P^{t} f-\bar{f}\right\| \leq\left(1-h^{2}[l]\right)^{\frac{t}{2 l}}\|f\|
$$

where we can use the Cheeger constant $h[l]$ for any even $l$.

Remark. Instead of considering the convergence in the norm $\|f \mid\|=$ $\sqrt{(f, f)_{\mu}}$, as in (9.1), one could also study stronger notions of convergence, e.g., the relative pointwise distance [33] or other measures of convergence as the mixing time $[20,33]$. All these quantities can be bounded from above in terms of the spectral radius of the transition probability operator $P$. Thus, the techniques developed in Sections 3 and 5 yield explicit bounds for the convergence of a random walk measured by any of these notions of convergence.

\section{Synchronization in coupled map lattices}

In this section, we present another application of our eigenvalue estimates.

We consider a coupled map lattice supported by a graph $\Gamma$, that is, a dynamical system updated at discrete times $t \in \mathbb{N}$ and of the form

$$
\left.x_{i}(t+1)=f\left(x_{i}(t)\right)+\frac{\epsilon}{d_{i}} \sum_{j} w_{i j}\left(f\left(x_{j}(t)\right)-f\left(x_{i}(t)\right)\right)\right),
$$

where $\epsilon \geq 0$ is the overall coupling strength and $w_{i j}$ is the strength of the interaction between unit $i$ and unit $j$. It was discovered by Kaneko [22] that the system (10.1) can (asymptotically) synchronize, i.e., $\left|x_{i}(t)-x_{j}(t)\right| \rightarrow 0$ for $t \rightarrow \infty$ and all $i, j$, even if the function $f$ displays chaotic behavior, i.e., 
its Lyapunov exponent $\mu(f)$ satisfies $^{2}$

$$
\mu(f)=\lim _{T \rightarrow \infty} \frac{1}{T} \sum_{t=0}^{T-1} \ln \left|f^{\prime}(s(t))\right|>0 .
$$

Here $s(t)$ is a synchronous solution, i.e., $x_{i}(t)=s(t)$ for all $i$.

More precisely, system (10.1) synchronizes under suitable conditions that will depend on $\mu(f), \epsilon$, and the properties of $\Gamma$. In particular, we have the following criterion for the asymptotic stability of a synchronized state (which, when fulfilled, implies that (10.1) will asymptotically synchronize when its initial values are sufficiently close to that state).

Theorem 10.1 [21]. A synchronized state $s(t)$ of the coupled map lattice (10.1) is asymptotically stable if

$$
\frac{1-e^{-\mu(f)}}{\lambda_{1}}<\epsilon<\frac{1+e^{-\mu(f)}}{\lambda_{N-1}} .
$$

Thus, there exists a range of values of $\epsilon$ for which we have asymptotic stability if

$$
\frac{\lambda_{N-1}}{\lambda_{1}}<\frac{e^{\mu(f)}+1}{e^{\mu(f)}-1} \quad \text { and } \quad \mu(f)>0
$$

or

$$
\frac{\lambda_{N-1}}{\lambda_{1}}>\frac{e^{\mu(f)}+1}{e^{\mu(f)}-1} \quad \text { and } \quad \mu(f)<0
$$

The non-trivial case here is, of course, the one where $\mu(f)>0$.

Thus, we can determine conditions under which system (10.1) synchronizes.

The main point of Theorem 10.1 is that the graph in question should be sufficiently different from both a disconnected graph (as characterized by $\lambda_{1}=0$ ) and a bipartite one (as characterized by $\lambda_{n-1}=2$ ). A disconnected graph cannot synchronize dynamics because its components do not interact.

${ }^{2}$ If $f$ preserves a "reasonable" good measure, then the Lyapunov exponent does not depend on the initial value $s(0)$ and a positive Lyapunov exponent implies positive topological entropy, which is usually used in the mathematical literature to characterize chaotic behavior [30]. In the following, we assume that $f$ preserves such a reasonable measure. 
Dynamics on a bipartite graph need not synchronize because the two classes can exchange their states every other period, that is, the bipartite graph can sustain non-synchronized period 2 oscillations. For a more general framework for synchronization of coupled dynamics, see [5].

In order to apply Theorem 10.1, we need to control the ratio $\frac{\lambda_{N-1}}{\lambda_{1}}$. So far, only suitable bounds, in terms of graph invariants, where known for $\lambda_{1}$. Using our estimates for the largest eigenvalue $\lambda_{N-1}$, we can now control the ratio $\frac{\lambda_{N-1}}{\lambda_{1}}$ in an appropriate way. In particular, the our results imply:

Corollary 10.1. For every graph we have:

$$
\begin{aligned}
\frac{\bar{h}}{h} & \leq \frac{\lambda_{N-1}}{\lambda_{1}} \\
& \leq \frac{\min \left[\min _{l \in \mathbb{N}, \text { even }} 1+\left(1-h[l]^{2}\right)^{\frac{1}{2 l}}, \min _{l \in \mathbb{N}, \text { odd }} 1+\left(1-(1-\bar{h}[l])^{2}\right)^{\frac{1}{2 l}}\right]}{\max _{l \in \mathbb{N}}\left(1-\left(1-h[l]^{2}\right)^{\frac{1}{2 l}}\right)}
\end{aligned}
$$

\section{Acknowledgments}

FB wants to thank Matthias Keller and Daniel Lenz for stimulating discussions during his stay in Jena.

\section{References}

[1] N. Alon and V. Milman, Isoperimetric inequalities for graphs, and superconcentrators, J. Combin. Theory Ser. B 38 (1985), 73-88.

[2] N. Alon, Eigenvalues and expanders, Combinatorica 6 (1986), 83-96.

[3] A. Banerjee and J. Jost, On the spectrum of the normalized graph Laplacian, Linear Algebra Appl. 428 (2008), 3015-3022.

[4] F. Bauer, Normalized graph Laplace operator for directed graphs, Linear Algebra Appl. 436 (2012), 4193-4222.

[5] F. Bauer, F. Atay and J. Jost, Synchronization in discrete-time networks with general pairwise coupling, Nonlinearity 22 (2009), $2333-2351$. 
[6] F. Bauer, J. Jost and S. Liu, Olliver-Ricci curvature and the spectrum of the normalized graph Laplace operator, to appear in Mathematical Research Letters, http://arxiv.org/abs/1105.3803.

[7] I. Chavel, Eigenvalues in Riemannian geometry, Academic Press, London, 1984.

[8] J. Cheeger, A lower bound for the smallest eigenvalue of the Laplace operator, in Problems in Analysis, ed. R.C. Gunning, 1970, 195-199.

[9] F.R.K. Chung, Laplacians of graphs and Cheeger inequalities in Combinatorics, Paul Erdös is Eighty, ed D. Miklós, V.T. Sós, T. Szönyi and János Bolyai, 2, Mathematical Society, Budapest, 1996 157-172.

[10] F.R.K. Chung, Spectral Graph Theory (CBMS Regional Conference Series in Mathematics, No. 92), American Mathematical Society, 1997.

[11] F.R.K. Chung, A. Grigor'yan and S.T. Yau, Upper bounds for eigenvalues of the discrete and continuous Laplace operators, Adv. Math. 117 (1996), 165-178.

[12] F.R.K. Chung and S.T. Yau, Eigenvalues of graphs and Sobolev inequalities, Combin. Probab. Comput. 4 (1995), 11-26.

[13] F.R.K. Chung and S.T. Yau, Eigenvalue inequalities for graphs and convex subgraphs, Comm. Geom. Anal. 5 (1998), 575-624.

[14] T. Coulhon and A. Grigor'yan, Random walks on graphs with regular volume growth, Geom. Funct. Anal. 8 (1998), 656-701.

[15] M. Desai and V. Rao A characterization of the smallest eigenvalue of a graph, J. Graph Theory 18(2) (1994), 181-194.

[16] P. Diaconis and D. Stroock, Geometric bounds for eigenvalues of Markov chains, Ann. Appl. Probab. 1(1) (1991), pp. 36-61.

[17] J. Dodziuk, Difference equations, isoperimetric inequality and transience of certain random walks, Trans. Amer. Math. Soc. 284 (1984), $787-794$.

[18] K. Fujiwara, Laplacians on rapidly branching trees, Duke Math. J. 83(1) (1996), 191-202.

[19] J. Fulman and E. Wilmer, Comparing eigenvalue bounds for Markov chains: When does Poincaré beat Cheeger?, Ann. Appl. Probab. 9 (1999), 1-13. 
[20] A. Grigoryan, Analysis on Graphs, Lecture Notes, University Bielefeld.

[21] J. Jost and M.P. Joy, Spectral properties and synchronization in coupled map lattices, Phys. Rev. E 65 (2001), 16201-16209.

[22] K. Kaneko, Clustering, coding, switching, hierarchical ordering, and control in a network of chaotic elements, Physica D 41 (1990), 137-172.

[23] M. Keller, The essential spectrum of the Laplacian on rapidly branching tessellations, Math. Ann. 346(1) (2010), 51-66.

[24] P. Li and S.T. Yau, Estimates of eigenvalues of a compact Riemannian manifold, AMS Proc. Symp. Pure Math. 36 (1980), 205-240.

[25] P. Li and S.T. Yau, On the parabolic kernel of the Schrödinger operator, Acta Math. 156 (1986), 153-201.

[26] R. Merris, Laplacian matrices of graphs: a survey, Linear Algebra Appl. 197-198 (1994), 143-176.

[27] B. Mohar, Isoperimetric numbers of graphs, J. Combin. Theory Ser. B 47 (1989), 274-291.

[28] Y. Ollivier, Ricci curvature of Markov chains on metric spaces, J. Funct. Anal. 256(3) (2009), 810-864.

[29] G. Polyá and S. Szegö, Isoperimetric inequalities in mathematical physics, Ann. Math. Stud. by Princeton University Press in London. (27) 1951.

[30] C. Robinson, Dynamical Systems, CRC Press, 1998.

[31] A. Sinclair and M. Jerrum, Approximating the permanent, SIAM J. Comput. 18 (1989), 1149-1178.

[32] A. Sinclair, Improved bounds for mixing rates of Markov chains and multicommodity flow, Combin., Probab. Comput. 1 (1992), 351-370.

[33] A. Sinclair, Algorithms for random gerneration 8 counting - a Markov chain approach, in Progress in theoretical computer science, Birkhäuser, 1993.

[34] T. Sunada, Discrete Geometric analysis, Proc. Symposia in Pure Mathematics, ed. P. Exner, J. P. Keating, P. Kuchment, T. Sunada, and A. Teplyaev, 77 (2008), 51-86. 
Max Planck Institute for Mathematics in the Sciences

INSELSTRASSE 22

D-04103 LEIPZIG

GERMANY

E-mail address: jost@mis.mpg.de

E-mail address: juergen.jost@mis.mpg.de

Received May 29, 2010 
